\title{
DOUBLY COMMUTING MIXED INVARIANT SUBSPACES IN THE POLYDISC
}

\author{
AMIT MAJI AND SANKAR T R
}

\begin{abstract}
We obtain a complete characterization for doubly commuting mixed invariant subspaces of the Hardy space over the unit polydisc. We say a closed subspace $\mathcal{Q}$ of $H^{2}\left(\mathbb{D}^{n}\right)$ is mixed invariant if $M_{z_{j}}(\mathcal{Q}) \subseteq \mathcal{Q}$ for $1 \leq j \leq k$ and $M_{z_{j}}^{*}(\mathcal{Q}) \subseteq \mathcal{Q}, k+1 \leq j \leq n$ for some integer $k \in\{1,2, \ldots, n-1\}$. We prove that a mixed invariant subspace $\mathcal{Q}$ of $H^{2}\left(\mathbb{D}^{n}\right)$ is doubly commuting if and only if

$$
\mathcal{Q}=\Theta H^{2}\left(\mathbb{D}^{k}\right) \otimes \mathcal{Q}_{\theta_{1}} \otimes \cdots \otimes \mathcal{Q}_{\theta_{n-k}},
$$

where $\Theta \in H^{\infty}\left(\mathbb{D}^{k}\right)$ is some inner function and $\mathcal{Q}_{\theta_{j}}$ is either a Jordan block $H^{2}(\mathbb{D}) \ominus \theta_{j} H^{2}(\mathbb{D})$ for some inner function $\theta_{j}$ or the Hardy space $H^{2}(\mathbb{D})$. Furthermore, an explicit representation for the commutant of an $n$-tuple of doubly commuting shifts as well as a representation for the commutant of a doubly commuting tuple of shifts and co-shifts are obtained. Finally, we discuss some concrete examples of mixed invariant subspaces.
\end{abstract}

\section{INTRODUCTION}

The celebrated Beurling theorem (see [4]) gives the structure of shift invariant subspaces in the Hardy space $H^{2}(\mathbb{D})$ over the unit disc: A non-zero closed subspace $\mathcal{S}$ of $H^{2}(\mathbb{D})$ is invariant for $M_{z}$ if and only if there exists an inner function $\theta \in H^{\infty}(\mathbb{D})$ such that

$$
\mathcal{S}=\theta H^{2}(\mathbb{D})
$$

In particular, we can represent $\mathcal{S}$ as

$$
\mathcal{S}=\bigoplus_{m=0}^{\infty} z^{m}(\mathcal{S} \ominus z \mathcal{S})
$$

A proper closed subspace $\mathcal{Q}$ of $H^{2}(\mathbb{D})$ is said to be a Jordan block of $H^{2}(\mathbb{D})$ if $\mathcal{Q}$ is $M_{z}^{*}$ invariant. Now $\mathcal{Q}$ is $M_{z}^{*}$-invariant if and only if $\mathcal{Q}^{\perp}$ is $M_{z}$-invariant. Thus by Beurling's theorem, there exists an inner function $\theta \in H^{\infty}(\mathbb{D})$ such that $\mathcal{Q}^{\perp}=\theta H^{2}(\mathbb{D})$. Therefore the Jordan block of $H^{2}(\mathbb{D})$ is determined by $\theta$ and is given by $\mathcal{Q}_{\theta}=H^{2}(\mathbb{D}) \ominus \theta H^{2}(\mathbb{D})$.

Now we are going to define Jordan block of the Hardy space over the unit polydisc. It is well known that the $n$-tuple of multiplication operators $\left(M_{z_{1}}, \ldots, M_{z_{n}}\right)$ on the Hardy space $H^{2}\left(\mathbb{D}^{n}\right), n>1$ over the unit polydisc is doubly commuting. A proper closed subspace $\mathcal{Q}$ of $H^{2}\left(\mathbb{D}^{n}\right)$ is said to be a Jordan block of $H^{2}\left(\mathbb{D}^{n}\right), n>1$ if $\mathcal{Q}$ is invariant under the adjoint of $\left(M_{z_{1}}, \ldots, M_{z_{n}}\right)$ and the $n$-tuple $\left(\left.P_{\mathcal{Q}} M_{z_{1}}\right|_{\mathcal{Q}}, \ldots,\left.P_{\mathcal{Q}} M_{z_{n}}\right|_{\mathcal{Q}}\right)$ is doubly commuting. Firstly,

2010 Mathematics Subject Classification. 32A10, 32A35, 47A13, 47A15, 47A20, 47A45, 47A80, 46E20, 47B35, 30H05, 30H10.

Key words and phrases. Mixed invariant subspace, bounded analytic functions, doubly commuting, multipliers, Jordan block, Hardy space. 
Douglas and Yang (see [9], [10]) started the study of Jordan block of the Hardy space $H^{2}\left(\mathbb{D}^{2}\right)$ over the bidisc. After that, Izuchi, Nakazi and Seto [20] classified the Jordan block of $H^{2}\left(\mathbb{D}^{2}\right)$. Recently, J. Sarkar [25] has given a complete characterization of the Jordan block of $H^{2}\left(\mathbb{D}^{n}\right)$ : A subspace $\mathcal{Q}$ of $H^{2}\left(\mathbb{D}^{n}\right)$ is a Jordan block if and only if $\mathcal{Q}=\mathcal{Q}_{\theta_{1}} \otimes \cdots \otimes \mathcal{Q}_{\theta_{n}}$, where $\mathcal{Q}_{\theta_{i}}$ is a Jordan block of $H^{2}(\mathbb{D})$.

The structure of joint invariant subspaces in the Hardy space $H^{2}\left(\mathbb{D}^{2}\right)$ over the bidisc and in general the Hardy space over the polydisc $H^{2}\left(\mathbb{D}^{n}\right), n>1$ is very complex. That is because of the subtleties of theory of holomorphic functions in several variables as well as the difficulty associated with the structure of $n$-tuples, $n>1$, of commuting isometries on Hilbert spaces. There are several mathematicians like Agrawal, Clark, and Douglas [1], Ahern and Clark [2], Guo [11, 12], Guo, Sun, Zheng and Zhong [13], Rudin [26], Izuchi [15], Mandrekar [23] etc. (also see [17, 16, 18, 6] and the references therein) have studied the structure of joint invariant subspaces. The four operators $R_{z_{1}}, R_{z_{2}}, C_{z_{1}}$, and $C_{z_{2}}$ play an important role in finding the structure of (joint) invariant subspace $\mathcal{S}$ of $H^{2}\left(\mathbb{D}^{2}\right)$, where $R_{z_{1}}=\left.M_{z_{1}}\right|_{\mathcal{S}}$ and $R_{z_{2}}=\left.M_{z_{2}}\right|_{\mathcal{S}}$, $C_{z_{1}}=\left.P_{\mathcal{S}^{\perp}} M_{z_{1}}\right|_{\mathcal{S}^{\perp}}$, and $C_{z_{2}}=\left.P_{\mathcal{S}^{\perp}} M_{z_{2}}\right|_{\mathcal{S}^{\perp}}$. For example, Mandrekar [23] showed that if the commutator $\left[R_{z_{1}}, R_{z_{2}}^{*}\right]=0$, then $\mathcal{S}=\varphi H^{2}\left(\mathbb{D}^{2}\right)$ for some inner function $\varphi \in H^{\infty}\left(\mathbb{D}^{2}\right)$. Again if $\left[C_{z_{1}}, C_{z_{2}}^{*}\right]=0$ or $\operatorname{rank}\left[C_{z_{1}}, C_{z_{2}}^{*}\right]=1$, then the structure of $\mathcal{S}$ is known (see [20]). But for $\operatorname{rank}\left[C_{z_{1}}, C_{z_{2}}^{*}\right]>1$, the structure of $\mathcal{S}$ is not known. Motivated by the above fact, Izuchi et. al. (see [19]) firstly introduced the concept of mixed invariant subspaces of $H^{2}\left(\mathbb{D}^{2}\right)$ : A closed subspace $\mathcal{S}$ of $H^{2}\left(\mathbb{D}^{2}\right)$ is said to be mixed invariant if $M_{z_{1}}(\mathcal{S}) \subseteq \mathcal{S}$ and $M_{z_{2}}^{*}(\mathcal{S}) \subseteq \mathcal{S}$. Clearly, if $\mathcal{S}$ is joint $\left(M_{z_{1}}, M_{z_{2}}^{*}\right)$ invariant, then $\mathcal{S}^{\perp}=H^{2}\left(\mathbb{D}^{2}\right) \ominus \mathcal{S}$ is again a joint $\left(M_{z_{1}}^{*}, M_{z_{2}}\right)$ invariant. Thus a natural question arises in the setting of the Hardy space over the unit polydisc:

What is the structure of doubly commuting mixed invariant subspaces of $H^{2}\left(\mathbb{D}^{n}\right), n>1$ ? More precisely, if a proper closed subspace $\mathcal{Q}$ of $H^{2}\left(\mathbb{D}^{n}\right)$ for $n>1$ is invariant under $\left(M_{z_{1}}, \ldots, M_{z_{k}}, M_{z_{k+1}}^{*}, \ldots, M_{z_{n}}^{*}\right)$ and the $n$-tuple $\left(\left.P_{\mathcal{Q}} M_{z_{1}}\right|_{\mathcal{Q}}, \ldots,\left.P_{\mathcal{Q}} M_{z_{n}}\right|_{\mathcal{Q}}\right)$ is doubly commuting, then what is the explicit structure of $\mathcal{Q}$ ?

In this paper we will give a complete characterization of doubly commuting mixed invariant subspaces of $H^{2}\left(\mathbb{D}^{n}\right), n>1$. We also find an explicit representation for the commutant of an $n$-tuple of doubly commuting shifts. Moreover, a representation of a large class of bounded operators which intertwine with some doubly commuting shifts and co-shifts on the Hardy space over the polydisc is obtained.

This paper is organized as follows: In Section 2 we give some basic definitions and results on the Hardy space over the polydisc. In Section 3, we find an explicit representation for the commutant of an $n$-tuple of doubly commuting shifts and also a representation for the commutant of a doubly commuting tuple of shifts and co-shifts on the Hardy space over the polydisc. In Section 4, we completely classify doubly commuting mixed invariant subspaces of the Hardy spaces over the polydisc. Finally, we discuss some applications in Section 5.

\section{Preliminaries}

For $n \geq 1$, let $\mathbb{D}^{n}=\mathbb{D} \times \cdots \times \mathbb{D}$ be the open unit polydisc in $\mathbb{C}^{n}$. Throughout the paper we use the notation $\boldsymbol{z}$ for the $n$-tuple $\left(z_{1}, \ldots, z_{n}\right)$ in $\mathbb{C}^{n}$. Also for any multi-index $\boldsymbol{k}=\left(k_{1}, \ldots, k_{n}\right) \in \mathbb{Z}_{+}^{n}$ and $\boldsymbol{z} \in \mathbb{C}^{n}$, we write $\boldsymbol{z}^{\boldsymbol{k}}=z_{1}^{k_{1}} \cdots z_{n}^{k_{n}}$. Let $\mathcal{H}$ and $\mathcal{K}$ be two separable 
complex Hilbert spaces. The set of all bounded linear operators from $\mathcal{H}$ to $\mathcal{K}$ is denoted by $\mathcal{B}(\mathcal{H}, \mathcal{K})$. If $\mathcal{H}=\mathcal{K}$, then we shall denote it by $\mathcal{B}(\mathcal{H})$ instead of $\mathcal{B}(\mathcal{H}, \mathcal{H})$. A contraction $T$ on $\mathcal{H}$ (that is, $\|T h\| \leq\|h\|$ for all $h \in \mathcal{H}$ ) is said to be a pure contraction if $T^{* m} \rightarrow 0$ as $m \rightarrow \infty$ in the strong operator topology. Also an isometry $V$ on $\mathcal{H}$ (that is, $V^{*} V=I_{\mathcal{H}}$ ) is said to be pure or shift if $V^{* m} \rightarrow 0$ as $m \rightarrow \infty$ in the strong operator topology (see [14, 24]).

The Hardy space $H^{2}\left(\mathbb{D}^{n}\right)$ over the polydisc $\mathbb{D}^{n}$ is the Hilbert space of all holomorphic functions $f$ on $\mathbb{D}^{n}$ such that

$$
\|f\|_{H^{2}\left(\mathbb{D}^{n}\right)}=\left(\sup _{0 \leq r<1} \int_{\mathbb{T}^{n}}\left|f\left(r e^{i \theta_{1}}, \ldots, r e^{i \theta_{n}}\right)\right|^{2} d \theta\right)^{\frac{1}{2}}<\infty,
$$

where $d \theta$ is the normalized Lebesgue measure on the $n$-dimensional torus $\mathbb{T}^{n}$, the distinguished boundary of $\mathbb{D}^{n}$. It is a well known and important reproducing kernel Hilbert space corresponding to the Szegö kernel $\mathbb{S}_{n}$ on $\mathbb{D}^{n}$, where

$$
\mathbb{S}_{n}(\boldsymbol{z}, \boldsymbol{w})=\prod_{j=1}^{n}\left(1-z_{j} \bar{w}_{j}\right)^{-1} \quad\left(\boldsymbol{z}, \boldsymbol{w} \in \mathbb{D}^{n}\right)
$$

Then one can see that

$$
\mathbb{S}_{n}^{-1}(\boldsymbol{z}, \boldsymbol{w})=\sum_{0 \leq|\boldsymbol{k}| \leq n}(-1)^{|\boldsymbol{k}|} \boldsymbol{z}^{\boldsymbol{k}} \overline{\boldsymbol{w}}^{\boldsymbol{k}}
$$

where $|\boldsymbol{k}|=\sum_{j=1}^{n} k_{j}$ and $0 \leq k_{j} \leq 1$ for all $j=1, \ldots, n$.

We can extend this definition to a Hilbert space valued Hardy space also. Let $\mathcal{E}$ be a Hilbert space, and the $\mathcal{E}$-valued Hardy space over $\mathbb{D}^{n}$ be denoted by $H_{\mathcal{E}}^{2}\left(\mathbb{D}^{n}\right)$. Then $H_{\mathcal{E}}^{2}\left(\mathbb{D}^{n}\right)$ is the $\mathcal{E}$-valued reproducing kernel Hilbert space with the $\mathcal{B}(\mathcal{E})$-valued kernel function

$$
(\boldsymbol{z}, \boldsymbol{w}) \mapsto \mathbb{S}_{n}(\boldsymbol{z}, \boldsymbol{w}) I_{\mathcal{E}} \quad\left(\boldsymbol{z}, \boldsymbol{w} \in \mathbb{D}^{n}\right)
$$

In the sequel, we shall identify the vector valued Hardy space $H_{\mathcal{E}}^{2}\left(\mathbb{D}^{n}\right)$ with $H^{2}\left(\mathbb{D}^{n}\right) \otimes \mathcal{E}$ by the help of the canonical unitary $U: H_{\mathcal{E}}^{2}\left(\mathbb{D}^{n}\right) \rightarrow H^{2}\left(\mathbb{D}^{n}\right) \otimes \mathcal{E}$ defined by

$$
U\left(\boldsymbol{z}^{\boldsymbol{k}} \eta\right)=\boldsymbol{z}^{\boldsymbol{k}} \otimes \eta \quad\left(\boldsymbol{k} \in \mathbb{Z}_{+}^{n}, \eta \in \mathcal{E}\right) .
$$

One can easily observe that the Hardy space $H^{2}\left(\mathbb{D}^{n}\right), n>1$ over the unit polydisc can be identified via the unitary map $U: H^{2}\left(\mathbb{D}^{n}\right) \rightarrow H^{2}(\mathbb{D}) \otimes \cdots \otimes H^{2}(\mathbb{D})$, where

$$
U\left(z_{1}^{k_{1}} z_{2}^{k_{2}} \cdots z_{n}^{k_{n}}\right)=z^{k_{1}} \otimes z^{k_{2}} \otimes \cdots \otimes z^{k_{n}},
$$

with the $n$-fold Hilbert space tensor product of the Hardy space $H^{2}(\mathbb{D})$ on the unit disc. Also we can realize $H^{2}(\mathbb{D}) \otimes \cdots \otimes H^{2}(\mathbb{D})$ as the vector-valued Hardy space $H_{H^{2}\left(\mathbb{D}^{n-1}\right)}^{2}(\mathbb{D})$ over the unit disc. This identification will be helpful to understand the structure of invariant subspaces of $H^{2}\left(\mathbb{D}^{n}\right)$.

Let $\left(M_{z_{1}}, \ldots, M_{z_{n}}\right)$ denote the $n$-tuple of multiplication operators on $H_{\mathcal{E}}^{2}\left(\mathbb{D}^{n}\right)$ by the coordinate functions $\left\{z_{j}\right\}_{j=1}^{n}$, that is,

$$
\left(M_{z_{j}} f\right)(\boldsymbol{w})=w_{j} f(\boldsymbol{w}),
$$

for all $f \in H_{\mathcal{E}}^{2}\left(\mathbb{D}^{n}\right), \boldsymbol{w} \in \mathbb{D}^{n}$ and $j=1, \ldots, n$. It is easy to see that $\left(M_{z_{1}}, \ldots, M_{z_{n}}\right)$ is an $n$-tuple of doubly commuting shifts on $H_{\mathcal{E}}^{2}\left(\mathbb{D}^{n}\right)$. Evidently, the shift $M_{z_{j}}$ on $H_{\mathcal{E}}^{2}\left(\mathbb{D}^{n}\right)$ can be 
identified with $M_{z_{j}} \otimes I_{\mathcal{E}}$ on $H^{2}\left(\mathbb{D}^{n}\right) \otimes \mathcal{E}$ for $j=1, \ldots, n$. This canonical identification will be used throughout the paper. We recall that a closed subspace $\mathcal{S}$ of $H_{\mathcal{E}}^{2}\left(\mathbb{D}^{n}\right)$ is called an invariant subspace for $\left(M_{z_{1}}, \ldots, M_{z_{n}}\right)$ if $M_{z_{j}}(\mathcal{S}) \subseteq \mathcal{S}$ for all $j=1, \ldots, n$. Let $I$ be any nonempty and proper subset of $\{1,2, \ldots, n\}$. We say a closed subspace $\mathcal{Q}$ of $H^{2}\left(\mathbb{D}^{n}\right)$ is mixed invariant if $M_{z_{j}}(\mathcal{Q}) \subseteq \mathcal{Q}$ for $j \in I$, and $M_{z_{j}}^{*}(\mathcal{Q}) \subseteq \mathcal{Q}$ for $j \in I^{c}$. Without loss of generality, we say that $\mathcal{Q}$ is mixed invariant under the multiplication operators $\left(M_{z_{1}}, \ldots, M_{z_{n}}\right)$ if $M_{z_{j}}(\mathcal{Q}) \subseteq \mathcal{Q}$ for $1 \leq j \leq k$ and $M_{z_{j}}^{*}(\mathcal{Q}) \subseteq \mathcal{Q}, k+1 \leq j \leq n$ for some integer $k \in\{1, \ldots, n-1\}$. In addition, $\mathcal{Q}$ is said to be doubly commuting mixed invariant if the $n$-tuple $\left(\left.P_{\mathcal{Q}} M_{z_{1}}\right|_{\mathcal{Q}}, \ldots,\left.P_{\mathcal{Q}} M_{z_{n}}\right|_{\mathcal{Q}}\right)$ is doubly commuting. We denote $H_{\mathcal{B}(\mathcal{E})}^{\infty}(\mathbb{D})$ as the Banach algebra of all $\mathcal{B}(\mathcal{E})$-valued bounded analytic functions on the open unit disc $\mathbb{D}$ (see [24]).

\section{COMMUTANT OF A DOUBLY COMMUTING SHIFTS}

In this section we obtain an explicit representation of the commutant of an $n$-tuple of doubly commuting shifts. We also find a representation of a large class of bounded operators which intertwine with shifts and adjoint of shifts, that is, co-shifts. As a by-product we give some examples of mixed invariant subspaces in the Hardy space over the polydisc.

Let $m, n$ be positive integers. For the sake of simplicity we denote $H^{2}\left(\mathbb{D}^{m}\right)$ by $H_{m}$. Here we use a similar technique used in our earlier paper (see [21]). We firstly denote $z_{j}$ as the coordinate function on $H^{2}\left(\mathbb{D}^{n}\right)$ for $j=1, \ldots, n$ and $w_{i}$ as the coordinate function on $H_{m}$ for $i=1, \ldots, m$. We then identify the Hardy space $H^{2}\left(\mathbb{D}^{n+m}\right)$ as $H_{m}$-valued Hardy space $H_{H_{m}}^{2}\left(\mathbb{D}^{n}\right)$ over $\mathbb{D}^{n}$ via canonical unitary. With this identification the multiplication operators $\left(M_{z_{1}}, \ldots, M_{z_{n}}, \ldots, M_{z_{n+m}}\right)$ on $H^{2}\left(\mathbb{D}^{n+m}\right)$ can be represented by $\left(M_{z_{1}}, \ldots, M_{z_{n}}, M_{\kappa_{1}}, \ldots, M_{\kappa_{m}}\right)$ on $H_{H m}^{2}\left(\mathbb{D}^{n}\right)$, where $\kappa_{i}$ is defined by

$$
\kappa_{i}(\boldsymbol{z})=M_{w_{i}}
$$

for all $\boldsymbol{z} \in \mathbb{D}^{n}$. It is evident that $\kappa_{i} \in H_{\mathcal{B}\left(H_{m}\right)}^{\infty}\left(\mathbb{D}^{n}\right)$ is a constant function and the multiplication operator $M_{\kappa_{i}}$ on $H_{H_{m}}^{2}\left(\mathbb{D}^{n}\right)$ is defined by

$$
M_{\kappa_{i}} f=\kappa_{i} f \quad\left(f \in H_{H_{m}}^{2}\left(\mathbb{D}^{n}\right)\right),
$$

is a shift on $H_{H_{m}}^{2}\left(\mathbb{D}^{n}\right)$ for all $i=1, \ldots, m$.

We now identify $H^{2}\left(\mathbb{D}^{n+m}\right)$ with $H^{2}\left(\mathbb{D}^{n}\right) \otimes H_{m}$ by the canonical unitary map $\hat{U}: H^{2}\left(\mathbb{D}^{n+m}\right) \rightarrow$ $H^{2}\left(\mathbb{D}^{n}\right) \otimes H_{m}$ defined by

$$
\hat{U}\left(z_{1}^{k_{1}} \cdots z_{n}^{k_{n}} \cdots z_{n+m}^{k_{n+m}}\right)=\left(z_{1}^{k_{1}} \cdots z_{n}^{k_{n}}\right) \otimes\left(w_{1}^{k_{n+1}} \cdots w_{m}^{k_{n+m}}\right),
$$

for all $k_{1}, \ldots, k_{n+m} \geq 0$. Then one can easily see that

$$
\hat{U} M_{z_{j}}=\left(M_{z_{j}} \otimes I_{H_{m}}\right) \hat{U} \quad(j=1, \ldots, n)
$$

and

$$
\hat{U} M_{z_{n+i}}=\left(I_{H^{2}\left(\mathbb{D}^{n}\right)} \otimes K_{i}\right) \hat{U} \quad(i=1, \ldots, m),
$$

where $K_{i}=M_{w_{i}}$. Thus the $(n+m)$-tuples $\left(M_{z_{1}}, \ldots, M_{z_{n}}, \ldots, M_{z_{n+m}}\right)$ on $H^{2}\left(\mathbb{D}^{n+m}\right)$ and $\left(M_{z_{1}} \otimes I_{H_{m}}, \ldots, M_{z_{n}} \otimes I_{H_{m}}, I_{H^{2}\left(\mathbb{D}^{n}\right)} \otimes K_{1}, \ldots, I_{H^{2}\left(\mathbb{D}^{n}\right)} \otimes K_{m}\right)$ on $H^{2}\left(\mathbb{D}^{n}\right) \otimes H_{m}$ are unitarily equivalent. 
We now define a unitary map $\tilde{U}: H^{2}\left(\mathbb{D}^{n}\right) \otimes H_{m} \rightarrow H_{H_{m}}^{2}\left(\mathbb{D}^{n}\right)$

$$
\tilde{U}\left(z_{1}^{k_{1}} \cdots z_{n}^{k_{n}} \otimes f\right)=z_{1}^{k_{1}} \cdots z_{n}^{k_{n}} f
$$

for all $k_{j} \geq 0$ and $f \in H_{m}$. Then

$$
\tilde{U}\left(M_{z_{j}} \otimes I_{H_{m}}\right)=M_{z_{j}} \tilde{U} \quad(j=1, \ldots, n) .
$$

For $\kappa_{i} \in H_{\mathcal{B}\left(H_{m}\right)}^{\infty}\left(\mathbb{D}^{n}\right)$, and each operator $M_{\kappa_{i}}: H_{H_{m}}^{2}\left(\mathbb{D}^{n}\right) \rightarrow H_{H_{m}}^{2}\left(\mathbb{D}^{n}\right)$ defined by

$$
\left(M_{\kappa_{i}}\left(z_{1}^{k_{1}} \cdots z_{n}^{k_{n}} f\right)\right)(\boldsymbol{x})=x_{1}^{k_{1}} \cdots x_{n}^{k_{n}}\left(K_{i} f\right),
$$

for all $k_{j} \geq 0, f \in H_{m}$ and $\boldsymbol{x}=\left(x_{1}, \ldots, x_{n}\right) \in \mathbb{D}^{n}$, is a shift on $H_{H_{m}}^{2}\left(\mathbb{D}^{n}\right)$. It also follows that

$$
\tilde{U}\left(I_{H^{2}\left(\mathbb{D}^{n}\right)} \otimes K_{i}\right)=M_{\kappa_{i}} \tilde{U} .
$$

Therefore by setting $U=\tilde{U} \hat{U}$, we see that $U: H^{2}\left(\mathbb{D}^{n+m}\right) \rightarrow H_{H_{m}}^{2}\left(\mathbb{D}^{n}\right)$ is a unitary operator satisfying

and

$$
U M_{z_{j}}=M_{z_{j}} U
$$

$$
U M_{z_{n+i}}=M_{\kappa_{i}} U
$$

for all $j=1, \ldots, n$ and $i=1, \ldots, m$.

To summarize this, we have the following:

Theorem 3.1. Let $m, n$ be positive integers and $H^{2}\left(\mathbb{D}^{n+m}\right)$ be the Hardy space over the unit polydisc $\mathbb{D}^{n+m}$. Then the $(n+m)$-tuples of multiplication operators $\left(M_{z_{1}}, \ldots, M_{z_{n}}, \ldots, M_{z_{n+m}}\right)$ on $H^{2}\left(\mathbb{D}^{n+m}\right)$ and $\left(M_{z_{1}}, \ldots, M_{z_{n}}, M_{\kappa_{1}}, \ldots, M_{\kappa_{m}}\right)$ on $H_{H_{m}}^{2}\left(\mathbb{D}^{n}\right)$ are unitarily equivalent, where $\kappa_{i} \in H_{\mathcal{B}\left(H_{m}\right)}^{\infty}\left(\mathbb{D}^{n}\right)$ is the constant function defined by

$$
\kappa_{i}(\boldsymbol{z})=M_{w_{i}} \in \mathcal{B}\left(H_{m}\right)
$$

for all $\boldsymbol{z} \in \mathbb{D}^{n}$ and $i=1, \ldots, m$.

We now return to the representation of the commutant for a tuple of doubly commuting shifts. Let $n$ be a fixed positive integer. Recall that for $\boldsymbol{z}=\left(z_{1}, \ldots, z_{n}\right) \in \mathbb{C}^{n}$ and for any multi-index $\boldsymbol{k}=\left(k_{1}, \ldots, k_{n}\right) \in \mathbb{Z}_{+}^{n}$, we write $\boldsymbol{z}^{\boldsymbol{k}}=z_{1}^{k_{1}} \cdots z_{n}^{k_{n}}$. Also for an $n$-tuple of commuting shifts $V=\left(V_{1}, \ldots, V_{n}\right), V^{\boldsymbol{k}}$ denotes $V_{1}^{k_{1}} \ldots V_{n}^{k_{n}}$. It is also well known (see [7]) that if $V=\left(V_{1}, \ldots, V_{n}\right)$ is an $n$-tuple of doubly commuting shifts on a Hilbert space $\mathcal{H}$, then

$$
\mathcal{H}=\oplus_{\boldsymbol{k} \in \mathbb{Z}_{+}^{n}} V^{\boldsymbol{k}} \mathcal{W}
$$

where $\mathcal{W}=\cap_{j=1}^{n} \operatorname{Ker}\left(V_{j}^{*}\right)$. We now define a map $\Pi: \mathcal{H} \rightarrow H_{\mathcal{W}}^{2}\left(\mathbb{D}^{n}\right)$ by

$$
\Pi\left(V_{1}^{k_{1}} \cdots V_{n}^{k_{n}} \eta\right)=z_{1}^{k_{1}} \cdots z_{n}^{k_{n}} \eta \quad\left(k_{j} \geq 0, \eta \in \mathcal{W}\right) .
$$

Then $\Pi$ is clearly a unitary. Moreover,

$$
\Pi V_{j}=M_{z_{j}} \Pi \text { for } j=1, \ldots, n .
$$

It is well known that if a bounded operator $X \in \mathcal{B}\left(H_{\mathcal{E}}^{2}\left(\mathbb{D}^{n}\right)\right)$ commutes with the multiplication operators $\left(M_{z_{1}}, \ldots, M_{z_{n}}\right)$ on $H_{\mathcal{E}}^{2}\left(\mathbb{D}^{n}\right)$, i.e., $X M_{z_{j}}=M_{z_{j}} X$ for all $1 \leq j \leq n$, then $X=M_{\Theta}$ for some $\Theta \in H_{\mathcal{B}(\mathcal{E})}^{\infty}\left(\mathbb{D}^{n}\right)$ (see [3] ). These are called analytic Toeplitz operators which is an 
important class of operators. Also the commutant of $V=\left(V_{1}, \ldots, V_{n}\right)$ on $\mathcal{H}$ can be represented as the commutant of $\left(M_{z_{1}}, \ldots, M_{z_{n}}\right)$ on $H_{\mathcal{W}}^{2}\left(\mathbb{D}^{n}\right)$. Hence the commutant of $V=\left(V_{1}, \ldots, V_{n}\right)$ on $\mathcal{H}$ can be written as the form $M_{\Phi}$ on $H_{\mathcal{W}}^{2}\left(\mathbb{D}^{n}\right)$, where $\Phi \in H_{\mathcal{B}(\mathcal{W})}^{\infty}\left(\mathbb{D}^{n}\right)$. The question is what is the explicit representation of $\Phi$ ? Here we have the following result.

Lemma 3.2. Let $\mathcal{H}$ be a Hilbert space and $V=\left(V_{1}, \ldots, V_{n}\right)$ be an $n$-tuple of doubly commuting shifts on $\mathcal{H}$. Let $T \in \mathcal{B}(\mathcal{H})$ and let $\Pi$ be the above unitary defined in (3.1). Then

$$
T V_{j}=V_{j} T \quad \text { for all } j=1, \ldots, n
$$

if and only if $\Pi T \Pi^{*}=M_{\Phi}$ for some $\Phi \in H_{\mathcal{B}(\mathcal{W})}^{\infty}\left(\mathbb{D}^{n}\right)$ and

$$
\Phi(\boldsymbol{z})=\left.P_{\mathcal{W}}\left(I_{\mathcal{H}}-z_{1} V_{1}^{*}\right)^{-1} \ldots\left(I_{\mathcal{H}}-z_{n} V_{n}^{*}\right)^{-1} T\right|_{\mathcal{W}}
$$

where $\boldsymbol{z}=\left(z_{1}, \ldots, z_{n}\right) \in \mathbb{D}^{n}, \mathcal{W}=\cap_{j=1}^{n} \operatorname{Ker}\left(V_{j}^{*}\right)$.

Proof. Let $h \in \mathcal{H}$. Then $h$ can be written as

$$
h=\sum_{k} V^{k} \eta_{k}
$$

for some $\eta_{\boldsymbol{k}} \in \mathcal{W}, \boldsymbol{k}=\left(k_{1}, \ldots, k_{n}\right) \in \mathbb{Z}_{+}^{n}$ (as $\left.\mathcal{H}=\oplus_{\boldsymbol{k} \in \mathbb{Z}_{+}^{n}} V^{\boldsymbol{k}} \mathcal{W}\right)$. Applying $P_{\mathcal{W}} V^{* \boldsymbol{m}}$ to both sides and since $\mathcal{W}=\cap_{j=1}^{n} \operatorname{Ker}\left(V_{j}^{*}\right)$, we have $\eta_{\boldsymbol{m}}=P_{\mathcal{W}} V^{* \boldsymbol{m}} h$ for all $\boldsymbol{m}=\left(m_{1}, \ldots, m_{n}\right) \in \mathbb{Z}_{+}^{n}$. Therefore, for any $h \in \mathcal{H}$,

$$
h=\sum_{k} V^{k} P_{\mathcal{W}} V^{* k} h
$$

Now let $T V_{j}=V_{j} T$ for all $j=1, \ldots, n$. Then there exists a bounded analytic function $\Phi \in H_{\mathcal{B}(\mathcal{W})}^{\infty}\left(\mathbb{D}^{n}\right)$ such that $\Pi T \Pi^{*}=M_{\Phi}$. For each $\boldsymbol{w}=\left(w_{1}, \ldots, w_{n}\right) \in \mathbb{D}^{n}$ and $\eta \in \mathcal{W}$ we have

$$
\begin{aligned}
\Phi(\boldsymbol{w}) \eta & =\left(M_{\Phi} \eta\right)(\boldsymbol{w}) \\
& =\left(\Pi T \Pi^{*} \eta\right)(\boldsymbol{w}) \\
& =(\Pi T \eta)(\boldsymbol{w}),
\end{aligned}
$$

as $\Pi^{*} \eta=\eta$. Using (3.2), we have

$$
T \eta=\sum_{\boldsymbol{k}} V^{\boldsymbol{k}} P_{\mathcal{W}} V^{* \boldsymbol{k}} T \eta
$$


it follows that

$$
\begin{aligned}
\Phi(\boldsymbol{w}) \eta & =\left(\Pi\left[\sum_{\boldsymbol{k}} V^{\boldsymbol{k}} P_{\mathcal{W}} V^{* \boldsymbol{k}} T \eta\right]\right)(\boldsymbol{w}) \\
& =\left[\sum_{\boldsymbol{k}} M_{\boldsymbol{z}}^{\boldsymbol{k}} P_{\mathcal{W}} V^{* \boldsymbol{k}} T \eta\right](\boldsymbol{w}) \\
& =\left[\sum_{\boldsymbol{k}} \boldsymbol{w}^{\boldsymbol{k}} P_{\mathcal{W}} V^{* \boldsymbol{k}} T \eta\right] \\
& =P_{\mathcal{W}}\left[\sum_{\boldsymbol{k}} \boldsymbol{w}^{\boldsymbol{k}} V^{* \boldsymbol{k}} T \eta\right] \\
& =P_{\mathcal{W}}\left(I_{\mathcal{H}}-w_{1} V_{1}^{*}\right)^{-1} \cdots\left(I_{\mathcal{H}}-w_{n} V_{n}^{*}\right)^{-1} T \eta
\end{aligned}
$$

Therefore

$$
\Phi(\boldsymbol{z})=\left.P_{\mathcal{W}}\left(I_{\mathcal{H}}-z_{1} V_{1}^{*}\right)^{-1} \cdots\left(I_{\mathcal{H}}-z_{n} V_{n}^{*}\right)^{-1} T\right|_{\mathcal{W}} \quad\left(\boldsymbol{z} \in \mathbb{D}^{n}\right)
$$

as required. We omit the sufficient part as it holds trivially.

This completes the proof.

REMARK 3.1. In the proof we have used the standard projection formula $I_{\mathcal{H}}=\mathrm{SOT}-\sum_{\boldsymbol{k}} V^{\boldsymbol{k}} P_{\mathcal{W}} V^{* \boldsymbol{k}}$.

Note that the function $\Phi$ defined in Lemma 3.2 is well defined and a $\mathcal{B}(\mathcal{W})$-valued holomorphic function in the unit polydisc $\mathbb{D}^{n}$ as $\left\|w_{j} V_{j}^{*}\right\|=\left|w_{j}\right|\left\|V_{j}\right\|<1$ for all $w_{j} \in \mathbb{D}$. Now if $T$ commutes with a shift $V_{1}$, then from the above result, we have the representation

$$
\Phi(z)=\left.P_{\mathcal{W}}\left(I_{\mathcal{H}}-z V_{1}^{*}\right)^{-1} T\right|_{\mathcal{W}} \quad\left(z \in \mathbb{D}, \mathcal{W}=\operatorname{Ker}\left(V_{1}^{*}\right)\right)
$$

which was obtained in our previous paper [22].

We are now in a position to find the explicit representation of the commutant of a tuple of doubly commuting shifts and co-shifts.

Theorem 3.3. Let $T$ be a bounded linear operator on $H_{H_{m}}^{2}\left(\mathbb{D}^{n}\right)$. Then $T$ satisfies

$$
T M_{z_{j}}=M_{z_{j}} T \quad \text { for } j=1, \ldots, n
$$

and

$$
T M_{\kappa_{i}}^{*}=M_{\kappa_{i}}^{*} T \quad \text { for } i=1, \ldots, m
$$

if and only if $T=M_{\Phi}$, where $\Phi \in H_{\mathcal{B}\left(H_{m}\right)}^{\infty}\left(\mathbb{D}^{n}\right)$ and

$$
\Phi(\boldsymbol{z})=\sum_{\boldsymbol{k} \in \mathbb{Z}_{+}^{n}} M_{\Theta_{k}}^{*} \boldsymbol{z}^{k},
$$

and

$$
\Theta_{\boldsymbol{k}}(\boldsymbol{w})=\sum_{\boldsymbol{l} \in \mathbb{Z}_{+}^{m}}\left\langle M_{\boldsymbol{z}}^{\boldsymbol{k}} \mathbf{1}, T \boldsymbol{w}^{\boldsymbol{l}}\right\rangle_{H_{H_{m}}^{2}\left(\mathbb{D}^{n}\right)} \boldsymbol{w}^{\boldsymbol{l}}
$$

for all $\boldsymbol{z}=\left(z_{1}, \ldots, z_{n}\right) \in \mathbb{D}^{n}, \boldsymbol{w}=\left(w_{1}, \ldots, w_{m}\right) \in \mathbb{D}^{m}$. 
Proof. Suppose that $T$ is a bounded linear operator on $H_{H_{m}}^{2}\left(\mathbb{D}^{n}\right)$ satisfying

$$
T M_{z_{j}}=M_{z_{j}} T \quad \text { for } j=1, \ldots, n
$$

and

$$
T M_{\kappa_{i}}^{*}=M_{\kappa_{i}}^{*} T \text { for } i=1, \ldots, m .
$$

From the first identity, we have $T=M_{\Phi}$, where $\Phi \in H_{\mathcal{B}\left(H_{m}\right)}^{\infty}\left(\mathbb{D}^{n}\right)$. Let

$$
\Phi(\boldsymbol{z})=\sum_{\boldsymbol{k}} T_{\boldsymbol{k}} \boldsymbol{z}^{\boldsymbol{k}} \quad\left(\boldsymbol{z} \in \mathbb{D}^{n}, T_{\boldsymbol{k}} \in \mathcal{B}\left(H_{m}\right)\right) .
$$

Now $H_{H_{m}}^{2}\left(\mathbb{D}^{n}\right)=\overline{\operatorname{span}}\left\{\boldsymbol{z}^{\boldsymbol{k}} \boldsymbol{w}^{\boldsymbol{l}}: \boldsymbol{k} \in \mathbb{Z}_{+}^{n}, \boldsymbol{l} \in \mathbb{Z}_{+}^{m}\right\}$. Since $T$ satisfies

$$
T M_{\kappa_{i}}^{*}=M_{\kappa_{i}}^{*} T \quad \text { for } i=1, \ldots, m \text {, }
$$

we have

$$
\begin{aligned}
T M_{\kappa_{i}}^{*}\left(\boldsymbol{w}^{\boldsymbol{r}}\right)=T M_{\kappa_{i}}^{*}\left(\boldsymbol{w}^{r} \boldsymbol{z}^{0}\right) & =\left(\sum_{\boldsymbol{k}} T_{\boldsymbol{k}} \boldsymbol{z}^{\boldsymbol{k}}\right) M_{w_{i}}^{*}\left(\boldsymbol{w}^{\boldsymbol{r}}\right) \\
& =\sum_{\boldsymbol{k}}\left(T_{\boldsymbol{k}} M_{w_{i}}^{*}\left(\boldsymbol{w}^{\boldsymbol{r}}\right)\right) \boldsymbol{z}^{\boldsymbol{k}}
\end{aligned}
$$

and

$$
M_{\kappa_{i}}^{*} T\left(\boldsymbol{w}^{r}\right)=M_{\kappa_{i}}^{*}\left(\sum_{\boldsymbol{k}} T_{\boldsymbol{k}} \boldsymbol{z}^{\boldsymbol{k}}\right)\left(\boldsymbol{w}^{\boldsymbol{r}}\right)=\sum_{\boldsymbol{k}}\left(M_{w_{i}}^{*} T_{\boldsymbol{k}}\left(\boldsymbol{w}^{\boldsymbol{r}}\right)\right) \boldsymbol{z}^{k}
$$

From the above we have,

$$
T_{\boldsymbol{k}} M_{w_{i}}^{*}\left(\boldsymbol{w}^{\boldsymbol{r}}\right)=M_{w_{i}}^{*} T_{\boldsymbol{k}}\left(\boldsymbol{w}^{\boldsymbol{r}}\right)
$$

for all $\boldsymbol{w}=\left(w_{1}, \ldots, w_{m}\right) \in \mathbb{D}^{m}$ and $\boldsymbol{k} \in \mathbb{Z}_{+}^{n}$. Therefore

$$
T_{\boldsymbol{k}} M_{w_{i}}^{*}=M_{w_{i}}^{*} T_{\boldsymbol{k}} \text { i.e., } M_{w_{i}} T_{\boldsymbol{k}}^{*}=T_{\boldsymbol{k}}^{*} M_{w_{i}}
$$

for all $i=1, \ldots, m$. Hence $T_{\boldsymbol{k}}^{*}=M_{\Theta_{\boldsymbol{k}}}$ for some $\Theta_{\boldsymbol{k}} \in H^{\infty}\left(\mathbb{D}^{m}\right)$ for all $\boldsymbol{k}$. Therefore

$$
\Phi(\boldsymbol{z})=\sum_{k} M_{\Theta_{k}}^{*} z^{k} \quad\left(z \in \mathbb{D}^{n}\right) .
$$

Now we will find $\Theta_{\boldsymbol{k}} \in H^{\infty}\left(\mathbb{D}^{m}\right)$ explicitly:

We know in view of Lemma 3.2 that

$$
\Phi(\boldsymbol{z})=\sum_{\boldsymbol{k}}\left(\left.P_{H_{m}} M_{\boldsymbol{z}}^{* \boldsymbol{k}} T\right|_{H_{m}}\right) \boldsymbol{z}^{\boldsymbol{k}} .
$$

Hence

$$
M_{\Theta_{\boldsymbol{k}}}^{*}=\left.P_{H_{m}} M_{\boldsymbol{z}}^{* \boldsymbol{k}} T\right|_{H_{m}},
$$


for all $\boldsymbol{k}$. We shall now calculate each $\Theta_{\boldsymbol{k}} \in H^{\infty}\left(\mathbb{D}^{m}\right)$. Suppose $\Theta_{\boldsymbol{k}}(\boldsymbol{w})=\sum_{\boldsymbol{l}} a_{\boldsymbol{l}} \boldsymbol{w}^{\boldsymbol{l}}$. Then

$$
\begin{aligned}
\left\langle\left(\sum_{l} a_{\boldsymbol{l}} \boldsymbol{w}^{\boldsymbol{l}}\right) \mathbf{1}, \boldsymbol{w}^{\boldsymbol{r}}\right\rangle_{H^{2}\left(\mathbb{D}^{m}\right)} & =\left\langle M_{\Theta_{\boldsymbol{k}}} \mathbf{1}, \boldsymbol{w}^{\boldsymbol{r}}\right\rangle_{H^{2}\left(\mathbb{D}^{m}\right)} \\
& =\left\langle\mathbf{1}, M_{\Theta_{\boldsymbol{k}}}^{*} \boldsymbol{w}^{\boldsymbol{r}}\right\rangle_{H^{2}\left(\mathbb{D}^{m}\right)} \\
& =\left\langle\mathbf{1}, P_{H_{m}} M_{\boldsymbol{z}}^{* \boldsymbol{k}} T \boldsymbol{w}^{\boldsymbol{r}}\right\rangle_{H^{2}\left(\mathbb{D}^{m}\right)} \\
& =\left\langle M_{\boldsymbol{z}}^{\boldsymbol{k}} \mathbf{1}, T \boldsymbol{w}^{\boldsymbol{r}}\right\rangle_{H_{H_{m}}^{2}\left(\mathbb{D}^{n}\right)} .
\end{aligned}
$$

Since $\left\langle\boldsymbol{w}^{l}, \boldsymbol{w}^{\boldsymbol{r}}\right\rangle=0$ for $\boldsymbol{l} \neq \boldsymbol{r}$ and $\boldsymbol{w}^{0}=\mathbf{1}$, a constant function on $H^{2}\left(\mathbb{D}^{m}\right)$, from the above we have $a_{\boldsymbol{r}}=\left\langle M_{\boldsymbol{z}}^{\boldsymbol{k}} \mathbf{1}, T \boldsymbol{w}^{\boldsymbol{r}}\right\rangle_{H_{H_{m}}^{2}\left(\mathbb{D}^{n}\right)}$ for all $\boldsymbol{r} \in \mathbb{Z}_{+}^{m}$. Thus

$$
\Phi(\boldsymbol{z})=\sum_{k} M_{\Theta_{k}}^{*} z^{k} \quad\left(z \in \mathbb{D}^{n}\right) .
$$

where

$$
\Theta_{\boldsymbol{k}}(\boldsymbol{w})=\sum_{l}\left\langle M_{z}^{k} \mathbf{1}, T \boldsymbol{w}^{l}\right\rangle_{H_{H_{m}}^{2}\left(\mathbb{D}^{n}\right)} \boldsymbol{w}^{l}
$$

The converse follows easily. This finishes the proof.

We know that if $T \in \mathcal{B}\left(H^{2}(\mathbb{D})\right)$ such that $T M_{z}=M_{z} T$, then $\overline{\operatorname{ran}}(T)$ is an $M_{z}$-invariant subspace of $H^{2}(\mathbb{D})$. In other words, for any $\varphi \in H^{\infty}(\mathbb{D}), \overline{r a n}\left(M_{\varphi}\right)$ is an $M_{z}$-invariant subspace of $H^{2}(\mathbb{D})$. Similarly let $T \in \mathcal{B}\left(H^{2}\left(\mathbb{D}^{n+m}\right)\right)$. Suppose that

$$
T M_{z_{j}}=M_{z_{j}} T \text { and } T M_{z_{n+i}}^{*}=M_{z_{n+i}}^{*} T,
$$

for $j=1, \ldots, n$ and $i=1, \ldots, m$. Then $\overline{\operatorname{ran}}(T)$ is invariant under $\left(M_{z_{1}}, \ldots, M_{z_{n}}, M_{z_{n+1}}^{*}, \ldots, M_{z_{n+m}}^{*}\right)$ on $H^{2}\left(\mathbb{D}^{n+m}\right)$. Thus from the above theorem, we can readily find some examples of mixed invariant subspaces.

Corollary 3.2. Let $T \in \mathcal{B}\left(H_{H_{m}}^{2}\left(\mathbb{D}^{n}\right)\right)$. Then $\overline{r a n}(T)$ is a mixed invariant subspace under $\left(M_{z_{1}}, \ldots, M_{z_{n}}, M_{\kappa_{1}}^{*}, \ldots, M_{\kappa_{m}}^{*}\right)$ if $T=M_{\Phi}$, where $\Phi \in H_{\mathcal{B}\left(H_{m}\right)}^{\infty}\left(\mathbb{D}^{n}\right)$ is given by

$$
\Phi(\boldsymbol{z})=\sum_{\boldsymbol{k} \in \mathbb{Z}_{+}^{n}} M_{\Theta_{k}}^{*} z^{k}
$$

and

$$
\Theta_{\boldsymbol{k}}(\boldsymbol{w})=\sum_{\boldsymbol{l} \in \mathbb{Z}_{+}^{m}}\left\langle M_{\boldsymbol{z}}^{\boldsymbol{k}} 1, T \boldsymbol{w}^{l}\right\rangle_{H_{H_{m}}^{2}\left(\mathbb{D}^{n}\right)} \boldsymbol{w}^{l}
$$

for all $\boldsymbol{z}=\left(z_{1}, \ldots, z_{n}\right) \in \mathbb{D}^{n}$ and $\boldsymbol{w}=\left(w_{1}, \ldots, w_{m}\right) \in \mathbb{D}^{m}$.

\section{Doubly COMmuting MiXed InVARIANt Subspaces}

In this section we obtain an explicit representation of doubly commuting mixed invariant subspaces. We start with a known result (see ([21]) for more details).

Lemma 4.1. Let $\mathcal{F}$ and $\mathcal{E}$ be Hilbert spaces and $M_{\Phi}: H_{\mathcal{F}}^{2}\left(\mathbb{D}^{n}\right) \rightarrow H_{\mathcal{E}}^{2}\left(\mathbb{D}^{n}\right)$ be an isometric multiplier. Then $\operatorname{dim}(\mathcal{F}) \leq \operatorname{dim}(\mathcal{E})$. 
The following result is required to prove our main results. The idea of the proof comes from a paper of Douglas and Foias [8]. This result may be known to the experts but here our proof is direct and it is useful as an independent result.

Lemma 4.2. Let $\mathcal{Q} \subseteq H_{\mathcal{E}}^{2}\left(\mathbb{D}^{n}\right)$ be an $M_{z_{j}}^{*}$-invariant subspace for all $j=1,2, \cdots, n$ and $T: \mathcal{Q} \rightarrow H^{2}\left(\mathbb{D}^{n}\right)$ be an isometry such that $\left.T M_{z_{j}}^{*}\right|_{\mathcal{Q}}=M_{z_{j}}^{*} T$ for all $j=1,2, \cdots, n$. Then there exists a one dimensional Hilbert space $\tilde{\mathcal{E}}$, a subspace of $\mathcal{E}$ such that $\mathcal{Q} \subseteq H_{\tilde{\mathcal{E}}}^{2}\left(\mathbb{D}^{n}\right)$ and a constant unitary multiplier $M_{\Theta}: H_{\tilde{\mathcal{E}}}^{2}\left(\mathbb{D}^{n}\right) \rightarrow H^{2}\left(\mathbb{D}^{n}\right)$ such that $T=\left.M_{\Theta}\right|_{\mathcal{Q}}$.

Proof. Since $\mathcal{Q}$ is an $M_{z_{j}}^{*}$-invariant subspace for all $j=1,2, \cdots, n$, the space

$$
\mathcal{M}=\overline{\operatorname{span}}\left\{\boldsymbol{z}^{\boldsymbol{k}} \mathcal{Q}: \boldsymbol{k} \in \mathbb{Z}_{+}^{n}\right\}
$$

is $M_{z_{j}}$-reducing subspace of $H_{\mathcal{E}}^{2}\left(\mathbb{D}^{n}\right)$ for all $j=1,2, \cdots, n$. We know that any $\left(M_{z_{1}}, M_{z_{2}}, \ldots, M_{z_{n}}\right)$ reducing subspace of $H_{\mathcal{E}}^{2}\left(\mathbb{D}^{n}\right)$ is of the form $H_{\tilde{\mathcal{E}}}^{2}\left(\mathbb{D}^{n}\right)$ for some closed subspace $\tilde{\mathcal{E}}$ of $\mathcal{E}$. Thus $\mathcal{M}=H_{\tilde{\mathcal{E}}}^{2}\left(\mathbb{D}^{n}\right)$. Firstly, our aim is to extend the operator $T: \mathcal{Q} \rightarrow H^{2}\left(\mathbb{D}^{n}\right)$ to $\tilde{T}: H_{\tilde{\mathcal{E}}}^{2}\left(\mathbb{D}^{n}\right) \rightarrow$ $H^{2}\left(\mathbb{D}^{n}\right)$ such that $\tilde{T}$ is an isometry and $M_{z_{j}}^{*} \tilde{T}=\tilde{T} M_{z_{j}}^{*}$ for all $j=1,2, \cdots, n$.

It is easy to see that the set $\left\{\boldsymbol{z}^{\boldsymbol{k}} \eta: \boldsymbol{k} \in \mathbb{Z}_{+}^{n}, \eta \in \mathcal{Q}\right\}$ is a total set in $H_{\tilde{\mathcal{E}}}^{2}\left(\mathbb{D}^{n}\right)$. Consider

$$
\mathcal{L}=\operatorname{span}\left\{\boldsymbol{z}^{\boldsymbol{k}} \eta: \boldsymbol{k} \in \mathbb{Z}_{+}^{n}, \eta \in \mathcal{Q}\right\}
$$

Let $F$ be a finite set in $\mathbb{Z}_{+}^{n}$. We define $\tilde{T}$ on $\mathcal{L}$ as

$$
\tilde{T}\left(\sum_{\boldsymbol{k} \in F} \alpha_{\boldsymbol{k}} \boldsymbol{z}^{\boldsymbol{k}} \eta_{\boldsymbol{k}}\right)=\sum_{\boldsymbol{k} \in F} \alpha_{\boldsymbol{k}} \boldsymbol{z}^{\boldsymbol{k}} T \eta_{\boldsymbol{k}}
$$

where $\alpha_{\boldsymbol{k}} \in \mathbb{C}$ and $\eta_{\boldsymbol{k}} \in \mathcal{Q}$. We shall firstly show that the map $\tilde{T}$ is well defined on $\mathcal{L}$. To do that we consider the following.

Let $F_{1}, F_{2}$ be two finite sets in $\mathbb{Z}_{+}^{n}$, and $\eta_{\boldsymbol{k}}, \zeta_{\boldsymbol{m}} \in \mathcal{Q}$. Then

$$
<\sum_{\boldsymbol{k} \in F_{1}} \alpha_{\boldsymbol{k}} \boldsymbol{z}^{\boldsymbol{k}} T \eta_{\boldsymbol{k}}, \sum_{\boldsymbol{m} \in F_{2}} \beta_{\boldsymbol{m}} \boldsymbol{z}^{\boldsymbol{m}} T \zeta_{\boldsymbol{m}}>=\sum_{\boldsymbol{k} \in F_{1}} \sum_{\boldsymbol{m} \in F_{2}} \alpha_{\boldsymbol{k}} \overline{\beta_{\boldsymbol{m}}}<\boldsymbol{z}^{\boldsymbol{k}} T \eta_{\boldsymbol{k}}, \boldsymbol{z}^{\boldsymbol{m}} T \zeta_{\boldsymbol{m}}>
$$

Now for each fixed $\boldsymbol{k} \in F_{1}$ and $\boldsymbol{m} \in F_{2}$, let $\boldsymbol{k}=\left(k_{1}, \ldots, k_{n}\right)$ and $\boldsymbol{m}=\left(m_{1}, \ldots, m_{n}\right)$. Consider $I=\left\{j \in\{1, \cdots, n\}: k_{j} \geq m_{j}\right\}$. Using the fact that $\left(M_{z_{1}}, \ldots, M_{z_{n}}\right)$ is a tuple of doubly 
commuting isometries and $T$ is an isometry, we obtain

$$
\begin{aligned}
<\boldsymbol{z}^{\boldsymbol{k}} T \eta_{\boldsymbol{k}}, \boldsymbol{z}^{\boldsymbol{m}} T \zeta_{\boldsymbol{m}}> & =<\prod_{j=1}^{n} M_{z_{j}}^{k_{j}} T \eta_{\boldsymbol{k}}, \prod_{j=1}^{n} M_{z_{j}}^{m_{j}} T \zeta_{\boldsymbol{m}}> \\
& =<\prod_{j \in I} M_{z_{j}}^{k_{j}-m_{j}} T \eta_{\boldsymbol{k}}, \prod_{j \in I^{c}} M_{z_{j}}^{m_{j}-k_{j}} T \zeta_{\boldsymbol{m}}> \\
& =<\prod_{j \in I^{c}} M_{z_{j}}^{*\left(m_{j}-k_{j}\right)} T \eta_{\boldsymbol{k}}, \prod_{j \in I} M_{z_{j}}^{*\left(k_{j}-m_{j}\right)} T \zeta_{\boldsymbol{m}}> \\
& =<T \prod_{j \in I^{c}} M_{z_{j}}^{*\left(m_{j}-k_{j}\right)} \eta_{\boldsymbol{k}}, T \prod_{j \in I} M_{z_{j}}^{*\left(k_{j}-m_{j}\right)} \zeta_{\boldsymbol{m}}> \\
& =<\prod_{j \in I^{c}} M_{z_{j}}^{*\left(m_{j}-k_{j}\right)} \eta_{\boldsymbol{k}}, \prod_{j \in I} M_{z_{j}}^{*\left(k_{j}-m_{j}\right)} \zeta_{\boldsymbol{m}}> \\
& =<\prod_{j \in I} M_{z_{j}}^{\left(k_{j}-m_{j}\right)} \eta_{\boldsymbol{k}}, \prod_{j \in I^{c}} M_{z_{j}}^{\left(m_{j}-k_{j}\right)} \zeta_{\boldsymbol{m}}> \\
& =<\prod_{j \in I} z_{j}^{m_{j}} \prod_{j \in I^{c}} z_{j}{ }_{j}\left(\prod_{j \in I} z_{j}^{\left(k_{j}-m_{j}\right)}\right) \eta_{\boldsymbol{k}}, \prod_{j \in I} z_{j}^{m_{j}} \prod_{j \in I^{c}} z_{j}^{k_{j}}\left(\prod_{j \in I} z_{j}{ }_{j}^{\left(m_{j}-k_{j}\right)}\right) \zeta_{\boldsymbol{m}}> \\
& =<\boldsymbol{z}^{\boldsymbol{k}} \eta_{\boldsymbol{k}}, \boldsymbol{z}^{\boldsymbol{m}} \zeta_{\boldsymbol{m}}>.
\end{aligned}
$$

Thus from the above equation (4.1), we have

$$
<\sum_{\boldsymbol{k} \in F_{1}} \alpha_{\boldsymbol{k}} z^{k} T \eta_{\boldsymbol{k}}, \sum_{\boldsymbol{m} \in F_{2}} \beta_{\boldsymbol{m}} z^{\boldsymbol{m}} T \zeta_{\boldsymbol{m}}>=<\sum_{\boldsymbol{k} \in F_{1}} \alpha_{\boldsymbol{k}} z^{k} \eta_{\boldsymbol{k}}, \sum_{\boldsymbol{m} \in F_{2}} \beta_{\boldsymbol{m}} z^{\boldsymbol{m}} \zeta_{\boldsymbol{m}}>.
$$

In particular for any finite set $F$ in $\mathbb{Z}_{+}^{n}$, we have

$$
\left\|\sum_{\boldsymbol{k} \in F} \alpha_{\boldsymbol{k}} \boldsymbol{z}^{\boldsymbol{k}} T \eta_{\boldsymbol{k}}\right\|^{2}=\left\|\sum_{\boldsymbol{k} \in F} \alpha_{\boldsymbol{k}} \boldsymbol{z}^{\boldsymbol{k}} \eta_{\boldsymbol{k}}\right\|^{2}
$$

Suppose that

$$
\sum_{k \in F_{1}} \alpha_{k} z^{k} \eta_{k}=\sum_{m \in F_{2}} \beta_{m} z^{m} \zeta_{m}
$$

Then from the above equality (4.2), it is easy to see that

$$
\left\|\sum_{\boldsymbol{k} \in F_{1}} \alpha_{\boldsymbol{k}} \boldsymbol{z}^{\boldsymbol{k}} T \eta_{\boldsymbol{k}}-\sum_{\boldsymbol{m} \in F_{2}} \beta_{\boldsymbol{m}} \boldsymbol{z}^{\boldsymbol{m}} T \zeta_{\boldsymbol{m}}\right\|^{2}=\left\|\sum_{\boldsymbol{k} \in F_{1}} \alpha_{\boldsymbol{k}} \boldsymbol{z}^{\boldsymbol{k}} \eta_{\boldsymbol{k}}-\sum_{\boldsymbol{m} \in F_{2}} \beta_{\boldsymbol{m}} \boldsymbol{z}^{\boldsymbol{m}} \zeta_{\boldsymbol{m}}\right\|^{2}=0 .
$$

Therefore

$$
\sum_{\boldsymbol{k} \in F_{1}} \alpha_{\boldsymbol{k}} \boldsymbol{z}^{\boldsymbol{k}} T \eta_{\boldsymbol{k}}=\sum_{\boldsymbol{m} \in F_{2}} \beta_{\boldsymbol{m}} \boldsymbol{z}^{\boldsymbol{m}} T \zeta_{\boldsymbol{m}} .
$$

Hence from the definition of $\tilde{T}$, we have

$$
\tilde{T}\left(\sum_{\boldsymbol{k} \in F_{1}} \alpha_{\boldsymbol{k}} \boldsymbol{z}^{\boldsymbol{k}} \eta_{\boldsymbol{k}}\right)=\tilde{T}\left(\sum_{\boldsymbol{m} \in F_{2}} \beta_{\boldsymbol{m}} \boldsymbol{z}^{\boldsymbol{m}} \zeta_{\boldsymbol{m}}\right) .
$$


This proves that $\tilde{T}$ is well defined on $\mathcal{L}$. From equation $(4.2)$, we can conclude that $\tilde{T}$ is a bounded as well as norm preserving linear operator on $\mathcal{L}$.

Now $\boldsymbol{k}=\left(k_{1}, \ldots, k_{n}\right) \in \mathbb{Z}_{+}^{n}, \eta \in \mathcal{Q}$, and for each $j=1,2, \cdots, n$, we have

$$
\tilde{T} M_{z_{j}}\left(\boldsymbol{z}^{\boldsymbol{k}} \eta\right)=\tilde{T}\left(z_{1}^{k_{1}} \cdots z_{j}^{k_{j}+1} \cdots z_{n}^{k_{n}} \eta\right)=\left(z_{1}^{k_{1}} \cdots z_{j}^{k_{j}+1} \cdots z_{n}^{k_{n}}\right) T \eta=z_{j} \boldsymbol{z}^{\boldsymbol{k}} T \eta=M_{z_{j}} \tilde{T}\left(\boldsymbol{z}^{\boldsymbol{k}} \eta\right) .
$$

Also if $k_{j} \geq 1$, then

$$
\tilde{T} M_{z_{j}}^{*}\left(\boldsymbol{z}^{\boldsymbol{k}} \eta\right)=\tilde{T}\left(z_{1}^{k_{1}} \cdots z_{j}^{k_{j}-1} \cdots z_{n}^{k_{n}} \eta\right)=\left(z_{1}^{k_{1}} \cdots z_{j}^{k_{j}-1} \cdots z_{n}^{k_{n}}\right) T \eta=M_{z_{j}}^{*}\left(\boldsymbol{z}^{\boldsymbol{k}} T \eta\right)=M_{z_{j}}^{*} \tilde{T}\left(\boldsymbol{z}^{\boldsymbol{k}} \eta\right) .
$$

If $k_{j}=0$, then

$$
\begin{aligned}
\tilde{T} M_{z_{j}}^{*}\left(\boldsymbol{z}^{\boldsymbol{k}} \eta\right)=\tilde{T}\left(z_{1}^{k_{1}} \cdots z_{j-1}^{k_{j-1}} z_{j+1}^{k_{j+1}} \cdots z_{n}^{k_{n}} M_{z_{j}}^{*} \eta\right) & =z_{1}^{k_{1}} \cdots z_{j-1}^{k_{j-1}} z_{j+1}^{k_{j+1}} \cdots z_{n}^{k_{n}}\left(T M_{z_{j}}^{*} \eta\right) \\
& =z_{1}^{k_{1}} \cdots z_{j-1}^{k_{j-1}} z_{j+1}^{k_{j+1}} \cdots z_{n}^{k_{n}}\left(M_{z_{j}}^{*} T \eta\right) \\
& =M_{z_{j}}^{*}\left(\boldsymbol{z}^{\boldsymbol{k}} T \eta\right) \\
& =M_{z_{j}}^{*} \tilde{T}\left(\boldsymbol{z}^{\boldsymbol{k}} \eta\right) .
\end{aligned}
$$

The above fact implies that

$$
\tilde{T} M_{z_{j}}=M_{z_{j}} \tilde{T} \quad \text { and }\left.\quad \tilde{T} M_{z_{j}}^{*}\right|_{\mathcal{L}}=M_{z_{j}}^{*} \tilde{T}
$$

for all $j=1,2, \cdots, n$. Now the norm preserving operator $\tilde{T}$ on $\mathcal{L}$ can be extended uniquely by continuity (again denoted by same $\tilde{T}$ ) to the closure of $\mathcal{L}\left(\right.$ i.e. $\overline{\mathcal{L}}=H_{\tilde{\mathcal{E}}}^{2}\left(\mathbb{D}^{n}\right)$ ) such that

$$
\tilde{T} M_{z_{j}}=M_{z_{j}} \tilde{T} \quad \text { and } \quad \tilde{T} M_{z_{j}}^{*}=M_{z_{j}}^{*} \tilde{T}
$$

for all $j=1,2, \cdots, n$. This imlplies that $\tilde{T}$ is a constant isometric multiplier from $H_{\tilde{\mathcal{E}}}^{2}\left(\mathbb{D}^{n}\right)$ to $H^{2}\left(\mathbb{D}^{n}\right)$. Therefore from Lemma 4.1, we have $\operatorname{dim}(\tilde{\mathcal{E}})=1$. Hence $\tilde{T}=M_{\Theta}$, where $\Theta(\boldsymbol{z})=\Theta(0)$ is a unitary from $\tilde{\mathcal{E}}$ to $\mathbb{C}$ for $\boldsymbol{z} \in \mathbb{D}^{n}$. Also

$$
T=\left.\tilde{T}\right|_{\mathcal{Q}}=\left.M_{\Theta}\right|_{\mathcal{Q}}
$$

This finishes the proof.

Theorem 4.3. Let $k \in\{1,2, \ldots, n-1\}$ be a fixed integer. Let $\mathcal{S}=\Theta H^{2}\left(\mathbb{D}^{k}\right) \otimes \mathcal{Q}_{\theta_{1}} \otimes \cdots \otimes \mathcal{Q}_{\theta n-k}$ be a joint $\left(M_{z_{1}}, \ldots, M_{z_{k}}, M_{z_{k+1}}^{*}, \ldots, M_{z_{n}}^{*}\right)$ invariant subspace of $H^{2}\left(\mathbb{D}^{n}\right)$, where $\Theta \in H^{\infty}\left(\mathbb{D}^{k}\right)$ is some inner function and $\mathcal{Q}_{\theta_{j}}$ is either a Jordan block or the Hardy space $H^{2}(\mathbb{D})$. Let

$$
V_{j}=\left.P_{\mathcal{S}} M_{z_{j}}\right|_{\mathcal{S}} \text { for } 1 \leq j \leq n .
$$

Then $\left(V_{1}, \cdots, V_{n}\right)$ is a doubly commuting tuple, where $V_{j}$ is a pure isometry for $1 \leq j \leq k$ and $V_{j}$ is a pure contraction for $k+1 \leq j \leq n$.

Proof. Suppose $\mathcal{S}=\Theta H^{2}\left(\mathbb{D}^{k}\right) \otimes \mathcal{Q}_{\theta_{1}} \otimes \cdots \otimes \mathcal{Q}_{\theta n-k} \subseteq H^{2}\left(\mathbb{D}^{n}\right)$ is a joint $\left(M_{z_{1}}, \ldots, M_{z_{k}}, M_{z_{k+1}}^{*}, \ldots, M_{z_{n}}^{*}\right)$ invariant subspace, where $\Theta \in H^{\infty}\left(\mathbb{D}^{k}\right)$ is some inner function and $\mathcal{Q}_{\theta_{j}}$ is either a Jordan block or the Hardy space $H^{2}(\mathbb{D})$. Clearly, $V_{j}=\left.P_{\mathcal{S}} M_{z_{j}}\right|_{\mathcal{S}}=\left.M_{z_{j}}\right|_{\mathcal{S}}$ for $1 \leq j \leq k$ is a pure isometry and $V_{j}=\left.P_{\mathcal{S}} M_{z_{j}}\right|_{\mathcal{S}}$ for $k+1 \leq j \leq n$ is a pure contraction.

Now $\Theta H^{2}\left(\mathbb{D}^{k}\right)$ is a doubly commuting invariant subspace for $\left(M_{z_{1}}, \ldots, M_{z_{k}}\right)$. Therefore $V_{i} V_{j}=V_{j} V_{i}$ and $V_{i} V_{j}^{*}=V_{j}^{*} V_{i}$ for $1 \leq i<j \leq k$. Again $\mathcal{Q}_{\theta_{1}} \otimes \cdots \otimes \mathcal{Q}_{\theta n-k}$ is a doubly 
commuting invariant subspace for $\left(M_{z_{k+1}}^{*}, \ldots, M_{z_{n}}^{*}\right)$. Thus $V_{i} V_{j}=V_{j} V_{i}$ and $V_{i} V_{j}^{*}=V_{j}^{*} V_{i}$ for $k+1 \leq i<j \leq n$. Also

$$
V_{i} V_{j}^{*}=\left.M_{z_{i}} M_{z_{j}}^{*}\right|_{\mathcal{S}}=\left.M_{z_{j}}^{*} M_{z_{i}}\right|_{\mathcal{S}}=V_{j}^{*} V_{i}
$$

for $1 \leq i \leq k$ and for $k+1 \leq j \leq n$.

To prove $V_{i} V_{j}=V_{j} V_{i}$ for $1 \leq i \leq k$ and for $k+1 \leq j \leq n$, we use the standard result of the tensor product of Hilbert spaces: Let $\mathcal{H}_{1}, \mathcal{H}_{2} \subseteq \mathcal{H}$ and $h_{1}, h_{2} \in \mathcal{H}$. Then

$$
P_{\mathcal{H}_{1} \otimes \mathcal{H}_{2}}\left(h_{1} \otimes h_{2}\right)=P_{\mathcal{H}_{1}} h_{1} \otimes P_{\mathcal{H}_{2}} h_{2} \text {. }
$$

Let $f \in \Theta H^{2}\left(\mathbb{D}^{k}\right)$ and $g_{l} \in \mathcal{Q}_{\theta_{l}}$ for $l=1, \ldots, n-k$. Then

$$
\begin{aligned}
& V_{i} V_{k+l}\left(f \otimes g_{1} \otimes \cdots \otimes g_{l} \otimes \cdots \otimes g_{n-k}\right) \\
& =V_{i} P_{\Theta H^{2}\left(\mathbb{D}^{k}\right) \otimes \mathcal{Q}_{\theta_{1}} \otimes \cdots \otimes \mathcal{Q}_{\theta n-k}}\left(f \otimes g_{1} \otimes \cdots \otimes M_{z} g_{l} \otimes \cdots \otimes g_{n-k}\right) \\
& =V_{i}\left(f \otimes P_{\mathcal{Q}_{\theta_{1}} \otimes \cdots \otimes \mathcal{Q}_{\theta n-k}}\left(g_{1} \otimes \cdots \otimes z g_{l} \otimes \cdots \otimes g_{n-k}\right)\right) \\
& =M_{z_{i}}\left(f \otimes g_{1} \otimes \cdots \otimes P_{\mathcal{Q}_{\theta_{l}}} z g_{l} \otimes \cdots \otimes g_{n-k}\right) \\
& =z_{i} f \otimes g_{1} \otimes \cdots \otimes P_{\mathcal{Q}_{\theta_{l}}} z g_{l} \otimes \cdots \otimes g_{n-k} \\
& =P_{\Theta H^{2}\left(\mathbb{D}^{k}\right) \otimes \mathcal{Q}_{\theta_{1}} \otimes \cdots \otimes \mathcal{Q}_{\theta n-k}}\left(z_{i} f \otimes g_{1} \otimes \cdots \otimes z g_{l} \otimes \cdots \otimes g_{n-k}\right) \\
& =V_{k+l} V_{i}\left(f \otimes g_{1} \otimes \cdots \otimes g_{l} \otimes \cdots \otimes g_{n-k}\right) .
\end{aligned}
$$

This completes the proof.

We record the following known result on the analytic model for a doubly commuting $n$-tuple of operators (see [5]). Let $T=\left(T_{1}, \ldots, T_{n}\right)$ be a doubly commuting pure $n$-tuple of operators on $\mathcal{H}$. Define the defect operator for the $n$-tuple $T$ as

$$
D_{T^{*}}=\left(\prod_{j=1}^{n}\left(I_{\mathcal{H}}-T_{j} T_{j}^{*}\right)\right)^{\frac{1}{2}} .
$$

and

$$
\mathcal{D}_{T^{*}}=\overline{\operatorname{ran}}\left(D_{T^{*}}\right)
$$

Theorem 4.4. Let $T=\left(T_{1}, \ldots, T_{n}\right)$ be a doubly commuting pure $n$-tuple of operators on $\mathcal{H}$. Then $\left(M_{z_{1}}, \ldots, M_{z_{n}}\right)$ on $H_{\mathcal{D}_{T^{*}}}^{2}\left(\mathbb{D}^{n}\right)$ is the minimal isometric dilation of $T$. That is there exists a joint $\left(M_{z_{1}}^{*}, \ldots, M_{z_{n}}^{*}\right)$ invariant subspace $\mathcal{Q}$ of $H_{\mathcal{D}_{T^{*}}}^{2}\left(\mathbb{D}^{n}\right)$ such that

$$
\left.T_{j} \cong P_{\mathcal{Q}} M_{z_{j}}\right|_{\mathcal{Q}} \quad(\text { for all } j=1, \ldots, n)
$$

and

$$
H_{\mathcal{D}_{T^{*}}}^{2}\left(\mathbb{D}^{n}\right)=\overline{\operatorname{span}}\left\{\boldsymbol{z}^{\boldsymbol{k}} \mathcal{Q}: \boldsymbol{k} \in \mathbb{Z}_{+}^{n}\right\}
$$

Using the above theorem, we characterize a doubly commuting tuple of shifts and pure contractions.

Lemma 4.5. Let $k \in\{1,2, \ldots, n-1\}$ be a fixed integer. Let $\left(T_{1}, \ldots, T_{k}, T_{k+1}, \ldots, T_{n}\right)$ be an $n$-tuple of doubly commuting operators on $\mathcal{H}$ such that $T_{j}$ is shift for $1 \leq j \leq k$ and $T_{j}$ is a pure contraction for $k+1 \leq j \leq n$. Then $\left(T_{1}, \ldots, T_{k}, T_{k+1}, \ldots, T_{n}\right)$ on $\mathcal{H}$ is unitarily eqivalent 
to $\left(M_{z_{1}}, \ldots, M_{z_{k}}, I_{H^{2}\left(\mathbb{D}^{k}\right)} \otimes \tau_{1}, \ldots, I_{H^{2}\left(\mathbb{D}^{k}\right)} \otimes \tau_{n-k}\right)$ on $H_{\mathcal{W}}^{2}\left(\mathbb{D}^{k}\right)$, where $\mathcal{W}=\cap_{j=1}^{k} \operatorname{Ker}\left(T_{j}^{*}\right)$ and $\tau_{j}$ is a pure contraction on $\mathcal{W}$ for $1 \leq j \leq n-k$.

Proof. Consider the map $\Pi: \mathcal{H} \rightarrow H_{\mathcal{W}}^{2}\left(\mathbb{D}^{k}\right)$ defined by

$$
\Pi\left(\prod_{j=1}^{k} T_{j}^{m_{j}} \eta\right)=\prod_{j=1}^{k} z_{j}^{m_{j}} \eta
$$

where $\eta \in \mathcal{W}$ and $m_{j} \in \mathbb{Z}_{+}$. Then clearly $\Pi$ is unitary and $\Pi T_{j}=M_{z_{j}} \Pi$ for $1 \leq j \leq k$. Now we know that if $C \in \mathcal{B}\left(H_{\mathcal{W}}^{2}\left(\mathbb{D}^{k}\right)\right)$ such that $M_{z_{j}} C=C M_{z_{j}}$ and $M_{z_{j}}^{*} C=C M_{z_{j}}^{*}$ for $1 \leq j \leq k$, then $C=M_{\tau}=I_{H^{2}\left(\mathbb{D}^{k}\right)} \otimes \tau$, where $\tau \in \mathcal{B}(\mathcal{W})$. Moreover, if $C$ is pure then $\tau$ is pure. Thus

$$
\Pi T_{k+j} \Pi^{*}=I_{H^{2}\left(\mathbb{D}^{k}\right)} \otimes \tau_{j},
$$

where $\tau_{j} \in \mathcal{B}(\mathcal{W})$ is a pure contraction for $1 \leq j \leq n-k$. Since $T_{k+i} T_{k+j}=T_{k+j} T_{k+i}$ and $T_{k+i}^{*} T_{k+j}=T_{k+j} T_{k+i}^{*}$, it readily follows that

$$
\tau_{i} \tau_{j}=\tau_{j} \tau_{i} \text { and } \tau_{i}^{*} \tau_{j}=\tau_{j} \tau_{i}^{*}
$$

for $1 \leq i<j \leq n-k$.

Now we are in a position to state our main result.

Theorem 4.6. Let $\mathcal{S}$ be a closed subspace of $H^{2}\left(\mathbb{D}^{n}\right)$ and $k \in\{1, \ldots, n-1\}$ be a fixed integer. Let $\mathcal{S}$ be a joint $\left(M_{z_{1}}, \ldots, M_{z_{k}}, M_{z_{k+1}}^{*}, \ldots, M_{z_{n}}^{*}\right)$ invariant subspace and

$$
V_{j}=\left.P_{\mathcal{S}} M_{z_{j}}\right|_{\mathcal{S}} \quad \text { for } j=1, \ldots, n \text {. }
$$

If $\left(V_{1}, \ldots, V_{n}\right)$ is doubly commuting, then $\mathcal{S}=\Theta H^{2}\left(\mathbb{D}^{k}\right) \otimes \mathcal{Q}_{\theta_{1}} \otimes \cdots \otimes \mathcal{Q}_{\theta n-k}$ for some $\Theta \in$ $H^{\infty}\left(\mathbb{D}^{k}\right)$ inner function and $\mathcal{Q}_{\theta_{j}}$ either a Jordan block or the Hardy space $H^{2}(\mathbb{D})$ for $j=$ $1, \ldots, n-k$.

Proof. Since $\mathcal{S}$ is a joint $\left(M_{z_{1}}, \ldots, M_{z_{k}}, M_{z_{k+1}}^{*}, \ldots, M_{z_{n}}^{*}\right)$ invariant subspace of $H^{2}\left(\mathbb{D}^{n}\right)$,

$$
V_{j}=\left.P_{\mathcal{S}} M_{z_{j}}\right|_{\mathcal{S}}=\left.M_{z_{j}}\right|_{\mathcal{S}}
$$

for $j=1, \ldots, k$ is shift and

$$
V_{j}=\left.P_{\mathcal{S}} M_{z_{j}}\right|_{\mathcal{S}}
$$

for $j=k+1, \ldots, n$ is a pure contraction. Hence by the above Lemma 4.5, there exists a unitary $\Pi: \mathcal{S} \rightarrow H_{\mathcal{W}}^{2}\left(\mathbb{D}^{k}\right)$ defined by

$$
\Pi\left(\prod_{j=1}^{k} V_{j}^{m_{j}} \eta\right)=\prod_{j=1}^{k} z_{j}^{m_{j}} \eta \quad(\eta \in \mathcal{W})
$$

such that

$$
\begin{aligned}
\Pi V_{j} & =M_{z_{j}} \Pi \text { for } j=1, \ldots, k \\
\Pi V_{k+i} & =\left(I_{H^{2}\left(\mathbb{D}^{k}\right)} \otimes \tau_{i}\right) \Pi \text { for } i=1, \ldots, n-k
\end{aligned}
$$

where $\mathcal{W}=\cap_{j=1}^{k} \operatorname{Ker}\left(V_{j}^{*}\right)$ and $\tau_{i}$ is a pure contraction on $\mathcal{W}$ with

$$
\tau_{i} \tau_{j}=\tau_{j} \tau_{i} \text { and } \tau_{i}^{*} \tau_{j}=\tau_{j} \tau_{i}^{*}
$$


for $1 \leq i<j \leq n-k$. Now from Theorem 4.4, we have $\tau^{*}=\left(\tau_{1}^{*}, \ldots, \tau_{n-k}^{*}\right)$ on $\mathcal{W}$ is unitarily equivalent to $\left(\left.M_{w_{1}}^{*}\right|_{\mathcal{Q}}, \ldots,\left.M_{w_{n-k}}^{*}\right|_{\mathcal{Q}}\right)$, where $\mathcal{Q}$ is a joint $\left(M_{w_{1}}^{*}, \ldots, M_{w_{n-k}}^{*}\right)$ invariant subspace of $H_{\mathcal{D}_{\tau^{*}}}^{2}\left(\mathbb{D}^{n-k}\right)$ and $\mathcal{D}_{\tau^{*}}=\overline{r a n}\left(\prod_{i=1}^{n-k}\left(I_{\mathcal{W}}-\tau_{i} \tau_{i}^{*}\right)\right)^{\frac{1}{2}}$. Let $U: \mathcal{W} \rightarrow \mathcal{Q} \subseteq H_{\mathcal{D}_{\tau^{*}}}^{2}\left(\mathbb{D}^{n-k}\right)$ be a unitary such that

$$
U \tau_{i}^{*}=\left.M_{w_{i}}^{*}\right|_{\mathcal{Q}} U \text { for } i=1, \ldots, n-k .
$$

Thus we have a unitary map $I_{H^{2}\left(\mathbb{D}^{k}\right)} \otimes U^{*}: H^{2}\left(\mathbb{D}^{k}\right) \otimes \mathcal{Q} \rightarrow H^{2}\left(\mathbb{D}^{k}\right) \otimes \mathcal{W}$ such that

$$
\left(I_{H^{2}\left(\mathbb{D}^{k}\right)} \otimes U^{*}\right)\left(M_{z_{j}} \otimes I_{\mathcal{Q}}\right)=\left(M_{z_{j}} \otimes I_{\mathcal{W}}\right)\left(I_{H^{2}\left(\mathbb{D}^{k}\right)} \otimes U^{*}\right)
$$

and

$$
\left(I_{H^{2}\left(\mathbb{D}^{k}\right)} \otimes U^{*}\right)\left(\left.I_{H^{2}\left(\mathbb{D}^{k}\right)} \otimes M_{w_{i}}^{*}\right|_{\mathcal{Q}}\right)=\left(I_{H^{2}\left(\mathbb{D}^{k}\right)} \otimes \tau_{i}^{*}\right)\left(I_{H^{2}\left(\mathbb{D}^{k}\right)} \otimes U^{*}\right)
$$

for $j=1, \ldots, k$ and $i=1, \ldots, n-k$.

Let $\iota: \mathcal{S} \rightarrow H^{2}\left(\mathbb{D}^{k}\right) \otimes H^{2}\left(\mathbb{D}^{n-k}\right)$ be the inclusion map. Therefore we have an operator

$$
\tilde{\Pi}=\iota \circ \Pi^{*} \circ\left(I_{H^{2}\left(\mathbb{D}^{k}\right)} \otimes U^{*}\right): H^{2}\left(\mathbb{D}^{k}\right) \otimes \mathcal{Q} \rightarrow H^{2}\left(\mathbb{D}^{k}\right) \otimes H^{2}\left(\mathbb{D}^{n-k}\right)
$$

which is an isometry as each one is so and also satisfying

$$
\tilde{\Pi}\left(M_{z_{j}} \otimes I_{\mathcal{Q}}\right)=\left(M_{z_{j}} \otimes I_{\mathcal{W}}\right) \tilde{\Pi} \quad \text { for } j=1, \ldots, k
$$

and

$$
\tilde{\Pi}\left(\left.I_{H^{2}\left(\mathbb{D}^{k}\right)} \otimes M_{w_{i}}^{*}\right|_{\mathcal{Q}}\right)=\left(I_{H^{2}\left(\mathbb{D}^{k}\right)} \otimes M_{w_{i}}^{*}\right) \tilde{\Pi} \quad \text { for } i=1, \ldots, n-k .
$$

Then from the first equality, we have $\tilde{\Pi}$ is an inner multiplier, say,

$$
\tilde{\Pi}=M_{\Phi},
$$

for some $\Phi \in H_{\mathcal{B}\left(\mathcal{Q}, H^{2}\left(\mathbb{D}^{n-k}\right)\right)}^{\infty}\left(\mathbb{D}^{k}\right)$. Again the other equality gives

$$
\left.\Phi\left(e^{i t}\right) M_{w_{j}}^{*}\right|_{\mathcal{Q}}=M_{w_{j}}^{*} \Phi\left(e^{i t}\right), \quad j=1, \ldots, n-k .
$$

for all $t \in \mathbb{R}^{k}$ and $e^{i t}=e^{i t_{1}} \cdots e^{i t_{k}}$. Since $\Phi\left(e^{i t}\right)$ is isometry almost everywhere (a.e.) with respect to the Lebesgue measure on the $k$-dimensional torus $\mathbb{T}^{k}$, from the above Lemma 4.2,

$$
\Phi\left(e^{i t}\right): \mathcal{Q} \rightarrow H^{2}\left(\mathbb{D}^{n-k}\right) \quad\left(e^{i t} \in \mathbb{T}^{k} \text { a.e. }\right)
$$

is the restriction of a constant isometric multiplier from $H_{\tilde{\mathcal{E}}}^{2}\left(\mathbb{D}^{n-k}\right)$ to $H^{2}\left(\mathbb{D}^{n-k}\right)$, where $\tilde{\mathcal{E}}$ is a one-dimensional space. Since $\tilde{\mathcal{E}}$ and $\mathbb{C}$ are one dimensional spaces, the space of all constant multipliers in $H_{\mathcal{B}(\tilde{\varepsilon}, \mathbb{C})}^{\infty}\left(\mathbb{D}^{n-k}\right)$ is also one dimensional. Hence there exists a unitary multiplier $M_{X}: H_{\tilde{\mathcal{E}}}^{2}\left(\mathbb{D}^{n-k}\right) \rightarrow H^{2}\left(\mathbb{D}^{n-k}\right)$ such that

$$
\Phi\left(e^{i t}\right)=\left.\Theta\left(e^{i t}\right) M_{X}\right|_{\mathcal{Q}} \quad\left(\text { a.e. on } \mathbb{T}^{k}\right)
$$

for some scalar $\Theta\left(e^{i t}\right)$. Since $\Phi$ is analytic, $\Theta$ is also analytic on $\mathbb{D}^{k}$. Also $\left|\Theta\left(e^{i t}\right)\right|=1$ as $\left.M_{X}\right|_{\mathcal{Q}}$ and $\Phi\left(e^{i t}\right)$ are isometries a.e. on $\mathbb{T}^{k}$. Hence $\Theta \in H^{\infty}\left(\mathbb{D}^{k}\right)$ is an inner function. Thus the inner multiplier $M_{\Phi}: H_{\mathcal{Q}}^{2}\left(\mathbb{D}^{k}\right) \rightarrow H_{H^{2}\left(\mathbb{D}^{n-k}\right)}^{2}\left(\mathbb{D}^{k}\right)$ factors as $\left.M_{\Theta} \otimes M_{X}\right|_{\mathcal{Q}}$ from $H^{2}\left(\mathbb{D}^{k}\right) \otimes \mathcal{Q}$ to $H^{2}\left(\mathbb{D}^{k}\right) \otimes H^{2}\left(\mathbb{D}^{n-k}\right)$. Therefore

$$
\mathcal{S}=\operatorname{ran}\left(M_{\Phi}\right)=M_{\Phi} H_{\mathcal{Q}}^{2}=\Theta H^{2}\left(\mathbb{D}^{k}\right) \otimes M_{X}(\mathcal{Q}) .
$$


Since $M_{X}: H_{\tilde{\mathcal{E}}}^{2}\left(\mathbb{D}^{n-k}\right) \rightarrow H^{2}\left(\mathbb{D}^{n-k}\right)$ is a constant unitary, it intertwines with $M_{w_{i}}^{*}$ for $i=$ $1, \ldots, n-k$. Hence $M_{X}(\mathcal{Q})$ is a closed doubly commuting invariant subspace of $H^{2}\left(\mathbb{D}^{n-k}\right)$. Therefore from the result [25], $M_{X}(\mathcal{Q})$ is of the form

$$
M_{X}(\mathcal{Q})=\mathcal{Q}_{\theta_{1}} \otimes \cdots \otimes \mathcal{Q}_{\theta n-k}
$$

where $\mathcal{Q}_{\theta_{j}}$ is either a Jordan block or the Hardy space $H^{2}(\mathbb{D}), j=1, \ldots, n-k$. Thus

$$
\mathcal{S}=\Theta H^{2}\left(\mathbb{D}^{k}\right) \otimes \mathcal{Q}_{\theta_{1}} \otimes \cdots \otimes \mathcal{Q}_{\theta n-k}
$$

This finishes the proof.

\section{Some Applications}

In this section we give some concrete examples of mixed invariant subspaces.

Let $\mathbb{T}$ be the unit circle and $K(\cdot, z)$ be the Szegö kernel on $H^{2}(\mathbb{D})$. We consider the examples of mixed invariant subspaces in the Hardy space $H^{2}\left(\mathbb{D}^{2}\right)$ over the bidisc for the sake of simplicity. We can identify $H^{2}\left(\mathbb{D}^{2}\right)$ as $H^{2}(\mathbb{D}) \otimes H^{2}(\mathbb{D})$. Let $\left(\alpha_{n}\right) \subset \mathbb{D}$ be a sequence such that $\sum_{n=0}^{\infty} 1-\left|\alpha_{n}\right|^{2}<\infty$. Consider for $N \in \mathbb{N}$,

$$
\mathcal{Q}_{N}=\operatorname{span}\left\{K\left(\cdot, \alpha_{1}\right), \ldots, K\left(\cdot, \alpha_{N}\right)\right\}
$$

and

$$
\mathcal{S}_{N}=H^{2}(\mathbb{D}) \otimes \mathcal{Q}_{N}
$$

Then clearly $\mathcal{S}_{N}$ is invariant under $\left(M_{z} \otimes I_{H^{2}(\mathbb{D})}, I_{H^{2}(\mathbb{D})} \otimes M_{z}^{*}\right)$. Also

$$
\left(\mathcal{S}_{N} \ominus z_{1} \mathcal{S}_{N}\right)=\left(\mathcal{S}_{N} \ominus\left(M_{z} \otimes I_{H^{2}(\mathbb{D})}\right) \mathcal{S}_{N}\right)=\left(H^{2}(\mathbb{D}) \ominus z H^{2}(\mathbb{D})\right) \otimes \mathcal{Q}_{N}=\mathbb{C} \otimes \mathcal{Q}_{N} .
$$

Thus $\mathcal{S}_{N}$ is an example of a mixed invariant subspace with $\operatorname{dim}\left(\mathcal{S}_{N} \ominus z_{1} \mathcal{S}_{N}\right)=N$ for each $N \in \mathbb{N}$. Moreover, if we take

$$
\mathcal{Q}=\overline{\operatorname{span}}\left\{K\left(\cdot, \alpha_{i}\right): i \in \mathbb{N}\right\}
$$

then $\mathcal{S}=H^{2}(\mathbb{D}) \otimes \mathcal{Q}$ is a mixed invariant subspace with $\operatorname{dim}\left(\mathcal{S} \ominus z_{1} \mathcal{S}\right)=\infty$. Note that here $\mathcal{Q}$ is a proper subspace of $H^{2}(\mathbb{D})$ as $\prod_{j=1}^{\infty} \frac{z-\alpha_{j}}{1-\overline{\alpha_{j} z}} \in \mathcal{Q}^{\perp}=H^{2}(\mathbb{D}) \ominus \mathcal{Q}$.

In particular, we have an explicit representation of the Beurling-Lax-Halmos's inner function $\Theta$ and the mixed invariant subspace $\mathcal{S}$ of $H^{2}\left(\mathbb{D}^{n}\right)$, where $\mathcal{S} \ominus z_{1} \mathcal{S}$ is one dimensional.

Theorem 5.1. Let $\mathcal{S} \subseteq H_{H_{n}}^{2}(\mathbb{D})$ be a closed subspace, and let $\mathcal{W}=\mathcal{S} \ominus z \mathcal{S}$. Suppose $\mathcal{S}$ is invariant under $\left(M_{z}, M_{\kappa_{1}}^{*}, \ldots, M_{\kappa_{n}}^{*}\right)$. Then $\operatorname{dim}(\mathcal{W})=1$ if and only if

$$
\mathcal{S}=\Theta H^{2}(\mathbb{D})
$$

where $\Theta \in H_{\mathcal{B}\left(\mathbb{C}, H_{n}\right)}^{\infty}(\mathbb{D})$ is an inner multiplier given by

$$
\left.\Theta(z)=\psi(z) K\left(\cdot, \overline{\varphi_{1}(z)}, \ldots, \overline{\varphi_{n}(z)}\right)\right) \quad(z \in \mathbb{D}),
$$

$\varphi_{j}, \psi \in H^{\infty}(\mathbb{D})$ and $\left|\varphi_{j}(z)\right|<1$ for all $z \in \mathbb{D}$ and

$$
\left|\psi\left(e^{i \theta}\right)\right|^{2}=\prod_{j=1}^{n}\left(1-\left|\varphi_{j}\left(e^{i \theta}\right)\right|^{2}\right) \text { a.e. on } \mathbb{T} \text {. }
$$


Proof. Suppose that $\mathcal{S}$ is a closed subspace of $H_{H_{n}}^{2}(\mathbb{D})$ which is invariant under $\left(M_{z}, M_{\kappa_{1}}^{*}, \ldots, M_{\kappa_{n}}^{*}\right)$. Consider

$$
\begin{aligned}
V & =\left.M_{z}\right|_{\mathcal{S}} \quad \text { and } \\
V_{j} & =\left.P_{\mathcal{S}} M_{\kappa_{j}}\right|_{\mathcal{S}} \text { for } j=1, \ldots, n .
\end{aligned}
$$

Since $V$ is a shift and $V_{j}^{*}$ commute with $V$, the tuple $\left(V, V_{1}^{*}, \ldots, V_{n}^{*}\right)$ on $\mathcal{S}$ is unitarily equivalent to $\left(M_{z}, M_{\varphi_{1}}, \ldots, M_{\varphi_{n}}\right)$ on $H_{\mathcal{W}}^{2}(\mathbb{D})$, where $\mathcal{W}=\mathcal{S} \ominus z \mathcal{S}$ and from Remark 3.1,

$$
\varphi_{j}(w)=\left.P_{\mathcal{W}}\left(I_{\mathcal{S}}-w V^{*}\right)^{-1} V_{j}^{*}\right|_{\mathcal{W}} \quad(w \in \mathbb{D}, j=1, \ldots, n) .
$$

Since $\mathcal{S}$ is joint invariant under $\left(M_{z}, M_{\kappa_{1}}^{*}, \ldots, M_{\kappa_{n}}^{*}\right)$ and $\operatorname{dim}(\mathcal{W})=1$, using the result ([21], Theorem 3.2 ), we have an isometry

$$
M_{\Theta}: H^{2}(\mathbb{D}) \rightarrow H_{H_{n}}^{2}(\mathbb{D}) \text { such that } \mathcal{S}=\Theta H^{2}(\mathbb{D}),
$$

where $\Theta \in H_{\mathcal{B}\left(\mathbb{C}, H_{n}\right)}^{\infty}(\mathbb{D})$ is an inner multiplier and $M_{\kappa_{j}}^{*} M_{\Theta}=M_{\Theta} M_{\varphi_{j}}$ for some $\varphi_{j} \in H^{\infty}(\mathbb{D})$ for $1 \leq j \leq n$. Therefore, for each $z \in \mathbb{D}$

$$
M_{z_{j}}^{*} \Theta(z)=\Theta(z) \varphi_{j}(z)=\varphi_{j}(z) \Theta(z)
$$

for $1 \leq j \leq n$. This implies $\Theta(z) \cdot 1=\Theta(z) \in H^{2}\left(\mathbb{D}^{n}\right)$ is an eigenvector for the operator $M_{z_{j}}^{*}$ with eigenvalue $\varphi_{j}(z)$. Hence $\left|\varphi_{j}(z)\right|<1$ for $z \in \mathbb{D}, 1 \leq j \leq n$. That means for each $z \in \mathbb{D}$

$$
\Theta(z) \in \operatorname{ker}\left(M_{z_{j}}-\overline{\varphi_{j}(z)} I_{H^{2}\left(\mathbb{D}^{n}\right)}\right)^{*} \quad \text { for all } 1 \leq j \leq n .
$$

This implies $\Theta(z) \in \operatorname{span}\left\{K\left(\cdot,\left(\overline{\varphi_{1}(z)}, \ldots, \overline{\varphi_{n}(z)}\right)\right\}\right.$, where

$$
K\left(\cdot, \overline{\varphi_{1}(z)}, \ldots, \overline{\varphi_{n}(z)}\right)(\boldsymbol{w})=\prod_{j=1}^{n} \frac{1}{1-\varphi_{j}(z) w_{j}} \quad\left(\boldsymbol{w} \in \mathbb{D}^{n}\right) .
$$

Therefore

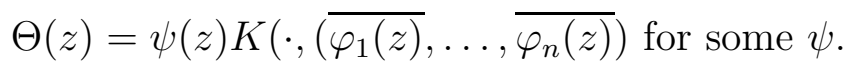

Using the fact that

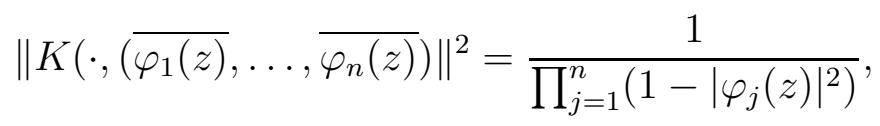

we have from the above identity

$$
|\psi(z)|^{2}=\|\Theta(z)\|^{2} \prod_{j=1}^{n}\left(1-\left|\varphi_{j}(z)\right|^{2}\right) .
$$

It is easy to see that $\psi \in H^{\infty}(\mathbb{D})$ as $|\psi(z)| \leq\|\Theta(z)\| \leq 1$ for $z \in \mathbb{D}$. Since $\Theta \in H_{\mathcal{B}\left(\mathbb{C}, H_{n}\right)}^{\infty}(\mathbb{D})$ is an inner multiplier, by taking the radial limit, we have

$$
\left|\psi\left(e^{i \theta}\right)\right|^{2}=\prod_{j=1}^{n}\left(1-\left|\varphi_{j}\left(e^{i \theta}\right)\right|^{2}\right) \text { a.e. on } \mathbb{T} \text {. }
$$

Also

$$
\mathcal{S}=\Theta H^{2}(\mathbb{D})
$$


where

$$
\Theta(z)=\psi(z) K\left(\cdot\left(\overline{\varphi_{1}(z)}, \ldots, \overline{\varphi_{n}(z)}\right)\right) \quad(z \in \mathbb{D}) .
$$

The converse follows easily.

This completes the proof.

The above result is a generalization of the result on the Hardy space $H^{2}\left(\mathbb{D}^{2}\right)$ over the bidisc by Izuchi et al.(see Theorem 3.2,[19]).

Acknowledgement: The authors are grateful to the anonymous reviewer for his/her critical and constructive reviews and suggestions that have substantially improved the presentation of the paper. The authors are also thankful to Prof. Jaydeb Sarkar for many fruitful discussions and his valuable comments. The first author's research work is supported by Faculty Initiation Grant (FIG scheme), IIT Roorkee (Ref. No: MAT/FIG/100820) and he also acknowledges Indian Statistical Institute, Bangalore Centre for warm hospitality.

\section{REFERENCES}

[1] O. Agrawal, D. Clark, and R. Douglas, Invariant subspaces in the polydisk, Pacific J. Math. 121 (1986), $1-11$.

[2] P. Ahern and D. Clark, Invariant subspaces and analytic continuation in several variables, J. Math. Mech. 19 (1970), 963-969.

[3] J. A. Ball, W. S. Li, D. Timotin, and T. T. Trent, A commutant lifting theorem on the polydisc, Indiana Univ. Math. J. 48 (1999), no. 2, 653-675.

[4] A. Beurling, On two problems concerning linear transformations in Hilbert space, Acta Math., 81 (1949), $239-255$.

[5] T. Bhattacharyya, E.K. Narayanan, J. Sarkar, Analytic model of doubly commuting contractions, Oper. Matrices 11 (1) (2017) 101-113.

[6] X. Chen and K. Guo, Analytic Hilbert modules, Chapman \& Hall/CRC Research Notes in Mathematics, 433. Chapman \& Hall/CRC, Boca Raton, FL, 2003.

[7] D. Popovici, A Wold-type decomposition for commuting isometric pairs, Proc. Amer. Math. Soc. 132 (2004), 2303-2314.

[8] R.G. Douglas, C. Foias, Uniqueness of multi-variate canonical models, Acta Sci. Math. 57 (1-4) (1993) 79-81.

[9] R. Douglas, R. Yang, Quotient Hardy modules, Houston J. Math. 24(1998), 507-517.

[10] R. Douglas, R. Yang, Operator theory in the Hardy space over the bidisk. I, Integral Equations Operator Theory 38(2000), 207-221.

[11] K. Guo, Defect operators for submodules of $H_{d}^{2}$, J. Reine Angew. Math. 573 (2004), 181-209.

[12] K. Guo, Algebraic reduction for Hardy submodules over polydisk algebras, J. Operator Theory 41 (1999), 127-138.

[13] K. Guo, S. Sun, D. Zheng and C. Zhong, Multiplication operators on the Bergman space via the Hardy space of the bidisk, J. Reine Angew. Math. 628 (2009), 129-168.

[14] P. Halmos, Shifts on Hilbert spaces, J. Reine Angew. Math. 208 (1961) 102-112.

[15] K. Izuchi, Unitary equivalence of invariant subspaces in the polydisk, Pacific Journal of Mathematics 130 (1987), 351-358. 
[16] K.J. Izuchi, K.H. Izuchi, Y. Izuchi, Blaschke products and the rank of backward shift invariant subspaces over the bidisk, J. Funct. Anal. 261(2011), 1457-1468.

[17] K.J. Izuchi, K.H. Izuchi, Y. Izuchi, Ranks of invariant subspaces of the Hardy space over the bidisk, J. Reine Angew. Math. 659(2011), 101-139.

[18] K.J. Izuchi, T. Nakazi, Backward shift invariant subspaces in the bidisc, Hokkaido Math. J. 3(2004), 247-254.

[19] Kei Ji Izuchi, Kou Hei Izuchi, Masatoshi Naito, Mixed invariant subspaces over the bidisk, Complex Anal. Oper. Theory 5 (2011), no. 4, 1003-1030.

[20] K. Izuchi, T. Nakazi, M. Seto, Backward shift invariant subspaces in the bidisc. II, J. Operator Theory 51(2004), 361-376.

[21] A. Maji, A. Mundayadan, J. Sarkar and Sankar T. R, Characterization of Invariant subspaces in the polydisc, J. Operator Theory 82 (2019), no. 2, 445-468

[22] A. Maji, J. Sarkar and Sankar T. R, Pairs of Commuting Isometries - I, Studia Mathematica, 248 (2019), no. 2, 171-189.

[23] V. Mandrekar, The validity of Beurling theorems in polydiscs, Proc. Amer. Math. Soc. 103 (1988), 145148.

[24] B. Sz.-Nagy and C. Foias, Harmonic analysis of operators on Hilbert space. North-Holland, AmsterdamLondon, 1970.

[25] J. SARKAR, Jordan blocks of $H^{2}\left(\mathbb{D}^{n}\right)$, J. Operator Theory 72 (2014), no. 2, 371-385.

[26] W. Rudin, Function Theory in Polydiscs, Benjamin, New York, 1969.

Indian Institute of Technology Roorkee, Department of Mathematics, Roorkee-247 667, UTTARAKHAND, INDIA

Email address: amit.maji@ma.iitr.ac.in, amit.iitmoł@gmail.com

Indian Statistical Institute, Statistics and Mathematics Unit, 8th Mile, Mysore Road, BANGALORE, 560059, INDIA

Email address: sankartr90@gmail.com 